\title{
Démocratie, famille et procédure
}

Ethnométhodologie d'un débat parlementaire syrien

Baudouin Dupret, Souhaïl Belhadj et Jean-Noël Ferrié

\section{CpenEdition}

Journals

Édition électronique

URL : http://journals.openedition.org/ress/181

DOI : $10.4000 /$ ress. 181

ISSN : 1663-4446

Éditeur

Librairie Droz

\section{Édition imprimée}

Date de publication : 1 novembre 2007

Pagination : 5-44

ISBN : 978-2-600-01207-2

ISSN : 0048-8046

Référence électronique

Baudouin Dupret, Souhaïl Belhadj et Jean-Noël Ferrié, " Démocratie, famille et procédure », Revue européenne des sciences sociales [En ligne], XLV-139 | 2007, mis en ligne le 01 novembre 2010, consulté le 20 avril 2019. URL : http://journals.openedition.org/ress/181 ; DOI : 10.4000/ress. 181 


\section{Baudouin DUPRET, Souhaïl BELHADJ, Jean-Noël FERRIÉ}

\section{DÉMOCRATIE, FAMILLE ET PROCÉDURE Ethnométhodologie d'un débat parlementaire syrien}

Fin 2003, les parlementaires syriens ont été invités à se prononcer sur un amendement du Code de statut personnel proposé par le gouvernement, en vertu duquel l'âge de la garde par leur mère des enfants mineurs, en cas de divorce, était étendu de 9 à 11 ans pour le garçon et de 11 à 13 ans pour la fille. En Commission des affaires constitutionnelles et législatives, la proposition fut modifiée, de telle sorte que le juge se voyait doter d'un pouvoir d'appréciation lui permettant d'étendre l'âge de la garde à 13 ans pour le garçon et à 15 pour la fille si l'intérêt de l'enfant l'exigeait. Ce projet de loi a été discuté au sein du Parlement syrien de manière soutenue. Composée de 250 membres élus au suffrage universel à la majorité simple à un tour, dont 167 appartiennent, conformément à la loi électorale, au Front national progressiste (coalition de partis sous la tutelle du Ba'th), l'Assemblée du Peuple abrite, depuis les années 1990, entre 70 et 80 indépendants. Cela signifie qu'en toute hypothèse, le FNP dispose d'une majorité supérieure aux deux tiers des membres, qui lui assure le contrôle de la révision de la Constitution et de la nomination du Président de la République. En outre, le Président de l'Assemblée a toujours été un membre du parti Ba 'th. Le débat sur l'amendement du droit de la famille fit intervenir un nombre important de membres de l'Assemblée, en ce compris des musulmans sunnites, des indépendants et des femmes. Dès le début du débat, un parlementaire sunnite indépendant, membre de la Commission des affaires constitutionnelles et législatives, proposa une troisième formule étendant d'office l'âge de la garde à 13 ans pour le garçon et 15 ans pour la fille, sans pouvoir d'appréciation du juge. Le débat suivit alors un cours tortueux et les questions relatives à la procédure et au règlement d'ordre intérieur survinrent avec une insistance toujours accrue, mettant aux prises quelques parlementaires et le Président de l'Assemblée dont la fonction régulatrice des débats s'est imposée manifestement. En fin de compte, c'est la troisième proposition (13 ans pour le garçon et 15 ans pour la fille, sans pouvoir d'appréciation du juge) qui fut adoptée par 87 voix pour et 75 voix contre.

En théorie politique, Hirschman est connu pour avoir opéré, sur la question des attitudes possibles face à des objets imposés, une typologie: la défection (exit), la prise de parole (voicing) ou le loyalisme (loyalty). Ce faisant, il cherchait à décrire les formes d'engagement des acteurs, sur des questions politiques, au travers desquelles ils marquent leur retrait, leur contestation ou leur adhésion. Sans qu'il s'agisse d'en nier la pertinence, cette typologie présente les inconvénients propres aux tentatives de construction d'idéaux-types et de formalisation du social, qui sont incapables de saisir la phénoménologie propre aux objets dont elles entendent se saisir. Autrement dit, nous nous retrouvons dotés d'intitulés de 
catégorie sans disposer du moindre accès aux modes de fonctionnement spécifiques de ce qu'elles cherchent à catégoriser. Notons qu'Hirschman est conscient de ce défaut, mais qu'il n'y apporte aucun remède.

Une des conséquences en est que les gens semblent être dans une attitude de soumission aux normes socio-politiques plutôt que dans une relation complexe par rapport à celles-ci. En somme, le travail théorique de catégorisation idéaletypique suscite au moins trois types de problèmes: il empêche de rendre compte du déroulement séquentiel des choses; il tend à fixer normativement le rattachement de telle ou telle instance à une catégorie, ce qui rend impossible de refléter la complexité des situations qui n'appartiennent jamais à une catégorie fixée à l'avance, mais s'inscrivent dans des contextes multiples; il produit un effacement radical du contexte dans lequel s'insèrent ces situations, faisant dès lors passer à la trappe tout ou partie des pertinences vers lesquelles les gens s'orientent, les hiérarchies qu'ils établissent contextuellement entre elles et les contraintes avec lesquelles ils sont amenés à composer.

Tout au contraire, nous entendons montrer comment, dans le contexte précis et contraignant (procéduralement et politiquement) d'un débat parlementaire syrien spécifique, sur une question de droit de la famille, des acteurs en chair et en os organisent collaborativement et processuellement leur adhésion, leur opposition ou leur abstention par rapport à l'objet de leur interaction, à savoir l'adoption d'un amendement au Code de statut personnel. Ce sont donc les attitudes des membres du Parlement syrien, face à une question particulière, que nous cherchons à saisir en action, dans leur phénoménologie propre, dans leur articulation dynamique et contextuelle. Plutôt que de postuler le rattachement de telle ou telle attitude à une catégorie, nous allons entrer dans le déploiement concret et situé du travail des parlementaires, dans son accomplissement même. A partir d'un matériau constitué de la retranscription verbatim du débat sur l'élévation de l'âge de la garde des enfants, nous serons en mesure d'analyser comment l'ordre est à la fois un accomplissement pratique et une ressource éventuelle. L'ordre est un accomplissement pratique, en ce sens que les gens procèdent en permanence en sorte d'être compréhensibles les uns par rapport aux autres, de se coordonner, d'organiser leurs accords et leurs désaccords, d'exprimer leurs divergences éventuelles dans un cadre général qu'ils reconnaissent et acceptent (solidarité sans consensus et solidarité négative). L'ordre est une ressource, par ailleurs, en ce sens que son énonciation formelle est régulièrement mobilisée et utilisée pour influencer le cours du débat, pour l'orienter, pour l'infléchir, pour parvenir à des fins spécifiques (produire une loi, discuter une politique, affirmer une position de pouvoir), pour s'opposer à la volonté d'autrui (le président du Parlement, par exemple), bref pour produire non seulement du débat parlementaire, mais aussi pour le produire dans un sens favorable aux intérêts que l'on se donne dans un cadre contextuellement et institutionnellement contraint.

Dans cet article, nous procéderons en trois temps. Il s'agira tout d'abord, en observant la double insertion du débat dans un site dialogique (le Parlement) et un réseau dialogique (l'ensemble des participants réels et virtuels au débat sur la famille) et sur la base d'une distinction entre arrière-plan de compréhension et contexte, de saisir le cadre politique et juridique de ce débat parlementaire. Il prend place dans une institution dont il faut décrire les modes de constitution et de fonctionnement, tout comme il porte sur un objet juridique, le droit de la famille, 
dont il importe de montrer les grandes lignes et les enjeux sociétaux. Deuxièmement, nous présenterons le débat dans son organisation générale, dans le détail de son déploiement et dans sa substance. Nous nous y intéresserons en tant qu'accomplissement pratique (l'ouverture de la discussion sur le projet d'amendement, le rôle du président, la répartition des tours de parole, le recours au vote, la décision d'une suspension de séance), tout en passant en revue les différentes interventions, leur articulation les unes aux autres, leurs registres argumentatifs et les jeux de catégorisation qui les sous-tendent. Dans un troisième temps, nous nous focaliserons sur la question de l'ordre en tant que ressource à la disposition des participants au débat. De ce point de vue, les usages du règlement intérieur de l'Assemblée du Peuple nous intéresseront particulièrement. Nous serons ainsi en mesure de montrer comment, loin de n'être qu'une norme déterminant strictement l'action, ce règlement peut être utilisé comme le moyen d'intervenir dans le cours du débat, de l'infléchir, de le faire traîner, de lui donner une orientation nouvelle. En outre, il s'agira pour nous de décrire comment, dans un contexte politique aux ressources et options limitées, l'argument d'ordre, légitime et légal, fonctionne comme un espace d'opportunité dans lequel les participants peuvent s'introduire en sorte d'exercer une influence (éventuellement efficace) sur le débat et, plus encore, sur son produit législatif.

\section{L'ARRIÈRE-PLAN POLITIQUE ET JURIDIQUE NÉCESSAIRE À L'INTELLIGIBILITÉ DU DÉBAT PARLEMENTAIRE}

Dans cette partie, nous entendons poser l'arrière-plan de compréhension nécessaire à l'analyse de ce débat parlementaire syrien. De ce point de vue, nous établissons une distinction épistémologique entre le contexte (les éléments de pertinence vers lesquels les participants s'orientent) et la compétence (l'arrièreplan de compréhension nécessaire, pour les participants comme pour l'analyste, à l'intelligibilité de l'activité parlementaire).

«Le discours parlementaire, d'un point de vue textuel, est fait d'une série de monologues abordant tous la même question. Mais la nature du discours n'est pas monologique mais dialogique » (Bayley 2004: 25). De notre point de vue, l'activité parlementaire est doublement dialogique. D'une part, les assemblées parlementaires sont des sites dialogiques. Par site, nous entendons que les nombreuses activités qui prennent place dans un seul et même lieu sont largement contraintes par cette inscription située. Le site est dialogique en ce sens que les propos et discours sont conçus comme des échanges entre participants autorisés, c'est-àdire les membres du Parlement. Les débats parlementaires sont des échanges de paroles organisés prenant place dans un tel site dialogique. Cela signifie que les échanges entre membres du Parlement suivent des règles spécifiques qui sont, pour partie, stipulées dans le Règlement intérieur de l'Assemblée et, pour une autre partie, inspirés des pratiques habituelles et des contraintes environnementales propres au discours institutionnel, aux échanges argumentatifs, aux débats politiques et aux caractéristiques spécifiques de ce lieu.

D'autre part, l'activité parlementaire, comme la plupart des activités sociales de nature politique, s'inscrit dans un réseau dialogique. A l'origine, cette notion vise à montrer que les événements médiatiques, tels que les programmes de 
télévision et de radio, les conférences de presse et les articles de journaux, fonctionnent en réseau, en ce sens qu'ils sont connectés interactionnellement, thématiquement et argumentativement, même si cette interconnexion dialogique est répartie dans le temps et l'espace (Nekvapil \& Leudar 2002; Leudar, Marsland, Nekvapil 2004). La notion a ensuite été étendue (Leudar \& Nekvapil 2007) pour intégrer des prises de parole à l'intérieur de l'enceinte parlementaire, où l'on observe que des interactions politiques en face-à-face, dans un site dialogique, s'articulent à des positions et des déclarations faites en-dehors de ses murs aussi bien qu'à des entités virtuelles (l'opinion publique, la société, la communauté internationale) dont la matérialité est postulée. Dans les termes de Bayley (2004: 24), «le discours parlementaire est composé d'une séquence de monologues qui sont intertextuellement et contratextuellement mêlés, dans la mesure où les parlementaires répondent à ce qui a été dit précédemment, non seulement au Parlement mais ailleurs ».

Quand donc nous parlons des Parlements et des activités qui s'y déroulent, il s'agit non pas de «carrefours de jeux spécifiques de déterminations historiques d'une sorte structurelle» (Carbó 2004: 303), ce qui relèverait d'un postulat indémontrable, mais de sites et réseaux dialogiques dont la nature empirique est attestée par les orientations propres aux participants aux débats qui s'y déroulent. Tel est le contexte de l'activité parlementaire.

Schématiquement, on peut affirmer que la notion de contexte s'étend d'une acception large (incluant des déterminants macro, meso et micro) à une définition étroite, le contexte étant alors restreint à ce qui est publiquement pertinent et procéduralement conséquentiel pour les participants. Nous penchons pour cette deuxième acception, tout en lui apportant quelques nuances par le biais de la distinction que nous opérons entre contexte de l'interaction et arrière-plan nécessaire à l'intelligibilité de celle-ci (ou compétence). En d'autres termes, nous considérons qu'il n'existe pas de contexte à postuler en-dehors de ce qui est disponible publiquement et observable empiriquement dans le cours des échanges discursifs (et parfois non discursifs) qui constituent et incarnent le processus d'adoption d'une loi. Cela n'empêche toutefois pas qu'aucun contexte disponible publiquement ne serait intelligible sans le savoir d'arrière-plan adéquat permettant aussi bien aux participants d'agir de manière compétente qu'à l'analyste de rendre compte et de faire sens de ce qui se passe dans ce contexte pour un participant compétent. Même la conception la plus restrictive du contexte dépend, pour rendre compte d'une activité, de compétences telles que le langage et la familiarité culturelle. Il ne s'agit toutefois que des conditions d'intelligibilité du contexte et non du contexte lui-même.

L'arrière-plan de compréhension du débat parlementaire sur l'élévation de l'âge de la garde des enfants est double. Il est fait, d'une part, de l'institution parlementaire syrienne, de son mode de constitution, de ses règles de fonctionnement, de la personnalité publiquement connue de ses membres, de son insertion dans la machine politique du pays.

L'exercice de l'activité législative par un conseil représentatif en Syrie remonte à 1919. Sa désignation, son mode de constitution et l'étendue de ses prérogatives a fluctué tout au long d'une histoire politique mouvementée. Tour à tour Assemblée parlementaire, Assemblée constituante, Assemblée nationale de la République Arabe Unie, Conseil national de la révolution et, enfin, Assemblée 
du Peuple, l'organe législatif représentatif n'a connu un rôle de premier plan que durant une courte période. On situe généralement aux années 1947-1951 et 19541958 (al-Khani, 2004), les phases de relatif pluralisme politique (en dépit de quatre coups de force) avec un Parlement qui désigne le Président de la République, comme en 1948, et qui est issu d'élections libres la plupart du temps.

De manière générale, l'instabilité politique chronique depuis 1949 n'a jamais permis qu'une législature parvienne à son terme légal. Quelques mois après un ultime coup d'Etat, fomenté par Hâfiz al-Asad en novembre 1970, l'institution parlementaire est rétablie par le décret $n^{\circ} 466$ : elle fait alors office d'assemblée constituante. La première session parlementaire de la nouvelle ère débute en juin 1973, soit quelques mois après la promulgation (février 1973) d'une constitution qui est toujours en vigueur actuellement. Depuis lors, huit législatures se sont succédé et la neuvième a débuté en 2007.

La Constitution de la République arabe syrienne de 1973 prévoit que le pouvoir législatif est dévolu à l'Assemblée du Peuple (Majlis al-sha'b). La chambre compte 250 sièges de députés élus pour quatre ans. Plus de la moitié de ces sièges (167) sont réservés au parti Ba'th et au front national progressiste, une coalition de huit partis qui lui sont affiliés. L'une des principales fonctions de l'Assemblée est d'approuver, certains diront enregistrer, le budget, les lois, les accords et traités internationaux engageant la souveraineté nationale et la population. La Constitution confère également à l'Assemblée du Peuple le pouvoir d'interpeller le gouvernement ou un ministre par le vote d'une motion de censure. Si cette dernière est adoptée, le cabinet ou le ministre en question doit présenter sa démission, bien que le gouvernement soit responsable devant le Président de la République.

De plus, le Parlement est la seule institution syrienne dont les membres soient élus à la faveur d'un scrutin national (à majorité simple à un tour). C'est aussi la seule assemblée élue qui joue un rôle dans le processus de désignation du chef de l'Etat. Les parlementaires sont sollicités dans la seconde étape d'une procédure d'investiture qui en compte trois: (1) proposition par le parti Ba'th du nom d'un candidat à l'Assemblée; (2) approbation de cette proposition par les députés; (3) soumission de la candidature unique au plébiscite. Si le prétendant à la présidence n'obtient pas la majorité absolue des suffrages exprimés, le Parlement doit représenter au plébiscite la candidature d'un autre (article 84).

Une fois élu, le Président de la République doit prêter serment devant les membres de l'Assemblée, de même qu'il doit leur remettre sa lettre de démission si les circonstances le réclament. En outre, seul le Parlement possède le pouvoir d'initier une procédure mettant en accusation le Président de la République, en vue de sa destitution (Art 91). L'Assemblée peut amender ou abroger les lois décrétées dans l'intervalle de ses sessions et doit approuver les décisions prises par le Président de déclarer la guerre ou de conclure la paix. Dans l'exercice de ses fonctions, le Président de la République peut dissoudre l'Assemblée du Peuple en vertu de l'article 107 et organiser de nouvelles élections législatives. En cas de vacance de la Présidence, ce n'est pas le président de l'Assemblée, mais le vice-Président de la République ou, en cas d'incapacité de ce dernier, le Premier ministre qui assure l'intérim. Depuis l'avènement de Hâfiz al-Asad et de son successeur, la dissolution n'a jamais été utilisée comme un instrument de pouvoir par la Présidence et les parlementaires ont toujours été au terme de leur mandat de quatre ans. 
C'est la loi électorale qui fixe les modalités du scrutin législatif. Ce dernier présente quelques particularités qu'il n'est pas utile de détailler ici. Néanmoins, il faut savoir que les candidatures doivent être déposées sur une des deux catégories suivantes: la catégorie A, réservée aux ouvriers et paysans, ou la catégorie B, destinée aux autres professions. Les candidats de la catégorie A peuvent s'inscrire sur la B, mais l'inverse est impossible. Le code électoral prévoit que le vote est direct et secret. Les candidatures sont examinées par des commissions présidées par le Gouverneur de province, un représentant des ouvriers et paysans et un représentant de la justice. Depuis 1990, il est, dans une plus large mesure, possible de se faire élire sans appartenir au Front national progressiste, en utilisant l'étiquette d'indépendant. L'Assemblée qui discute le projet de loi dont cet article traite compte 83 indépendants.

Globalement, les modalités de l'élection législative en Syrie répondent à deux impératifs. Le premier est d'assurer au Ba 'th, en tant qu'organisation partisane, sa prééminence, voire son hégémonie, au sein du système politique. Ce principe tire sa légitimité de l'article 8 de la Constitution selon lequel le parti Ba'th arabe socialiste est «le Parti dirigeant dans la Société et l'Etat». C'est ce qui explique qu'à chaque nouvelle législature, depuis 1973, l'Assemblée est composée d'une écrasante majorité de députés ba 'thistes. Le fait que la présidence du Parlement revienne à un homme politique issu de leurs rangs est une marque de cette hégémonie. Le second impératif est d'assurer la légitimation du régime. Dans la symbolique du pouvoir syrien, les masses sont à la source de la légitimité et elles doivent donner périodiquement leur aval à la mise en œuvre de la politique générale du pays. Ajoutons cependant qu'en dépit de l'attribution par la loi électorale de 167 sièges au Ba'th et aux partis subordonnés - suivant la proportion de 3/4 pour le premier et $1 / 4$ pour les autres -, les députés indépendants, certes adoubés par le régime, représentent des groupes d'intérêts susceptibles d'obtenir l'amendement de textes dans un sens non prévu par les caciques du Parti. En outre, les parlementaires ba 'thistes n'obéissent pas toujours à la discipline du Parti et s'affranchissent parfois des dogmes idéologiques.

L'essentiel du travail législatif revient théoriquement au Parlement. Le Président de la République peut toutefois faire opposition à certaines lois, par décret motivé, et il assume la totalité du pouvoir législatif dans l'intervalle des sessions et des législatures. En réalité, l'activité législative de la Présidence concurrence largement celle de l'Assemblée. Premièrement, parce que les vacances parlementaires sont très longues. Deuxièmement, parce que la constitution lui donne le pouvoir de décréter presque sans condition. De fait, les lois qui ont eu le plus d'impact sur la vie politique syrienne, depuis 2000 (élection de Bashshâr alAsad), sont de facture présidentielle. Troisièmement, parce que le processus électoral qui donne accès à la députation est étroitement contrôlé par la Présidence et par la direction du parti Ba'th, afin de s'assurer d'une Assemblée docile. En conséquence, le Parlement ne débat pas de ce qui relève de la réforme de l'Etat, des questions stratégiques ou de la transformation des structures économiques. Autrement dit, sa capacité à influer sur les «grandes options de la politique syrienne » est réduite. Ce régime est donc un régime présidentiel par excellence et le pouvoir exécutif connaît peu d'obstacles à son surcroît d'influence.

Malgré tout, la constitution fait de l'Assemblée du Peuple la seule institution limitative - formellement, potentiellement, mais aussi de fait - de l'extension du 
pouvoir présidentiel. Elle représente également une contrainte de type organique à l'orientation et au champ d'application de la politique en général, mais surtout des politiques publiques. Elle est, de facto, le pendant du gouvernement syrien en ce qui concerne l'initiative des lois, surtout celles touchant aux questions de morale et de société. A ce titre, le projet de loi à l'étude ici est un exemple de l'inscription de l'Assemblée du Peuple dans le complexe institutionnel et le jeu politique. A l'instigation de ce projet, on trouve le gouvernement et, plus précisément, le ministre de la Justice, M. Nizâr al-"Asasî, lui-même ancien député. Le Premier ministre lui a associé Ghadâ al-Jabî, ministre du Travail et des Affaires sociales. Le Cabinet souhaitait que Mme al-Jabî soutienne ce projet en raison du crédit dont elle bénéficiait auprès de la classe politique du pays et, particulièrement, auprès des députés, en tant qu'ancienne présidente de l'Union générale des femmes syriennes.

L'arrière-plan de compréhension du débat parlementaire sur l'élévation de l'âge de la garde des enfants est aussi fait du droit syrien de la famille, de ses textes et de ses institutions. Pour comprendre le débat sur l'élévation de l'âge de la garde, il convient donc également d'en saisir l'arrière-plan juridique.

Alors que la plupart des domaines du droit se sont progressivement autonomisés de la sharî‘ $a$ au cours du dix-neuvième siècle, le droit de la famille a suivi un schéma différent. Les tentatives de codification de ce qui se trouva bientôt appelé droit du statut personnel se multiplièrent. En 1917, l'Empire ottoman, s'inspirant de la doctrine hanéfite, promulgua un code complet en la matière.

La Syrie a adopté un Code de statut personnel s'appliquant à tous ses citoyens, musulmans ou non. Il fut promulgué le 7 septembre 1953 (loi $N^{\circ} 59$ de l’année 1953). Ce texte s'inscrit dans le mouvement dit de «révolution législative» qui se développa durant la brève présidence de Husnî al-Za‘îm (1949-1951), bien qu'il ait été promulgué au cours de la présidence d'Adîb al-Shishaklî (1951-1953). Le Code de statut personnel fut amendé par la loi N 34 de l'année 1975).

Parallèlement à la codification du droit syrien, le pouvoir judiciaire a été réorganisé et unifié en un système unique de cours et tribunaux. Les tribunaux de la sharî́a, intégrés au nouveau système, n'ont pas cessé pour autant de conserver une existence distincte. Les juridictions sont organisées à deux niveaux (première instance et appel), certains jugements pouvant faire l'objet d'un pourvoi devant la Cour de cassation. C'est la procédure ordinaire qui est suivie en matière de statut personnel.

Le statut personnel des Syriens est fonction de leur confession religieuse individuelle. Le décret-loi Nº 60 de l'année 1936, tel qu'amendé, définit les confessions reconnues en Syrie. Le Code de statut personnel, suivant en cela la traduction du fiqh, est divisé en six parties: mariage, divorce, filiation, capacité, donations, successions. Bien qu'il soit rédigé dans un langage abstrait et universel, le Code présuppose l'existence de lois autonomes pour chacune des 17 confessions reconnues. Autrement dit, il suppose que toutes les confessions suivent le même code, alors même que chacune suit sa propre loi. Le principe veut, en effet, que le Code de statut personnel s'applique à tous les Syriens (art. 306), sauf dans les cas stipulés aux articles 307 et 308. L'article 307 vise la communauté druze et interdit donc la polygamie, n'autorise pas la reprise de la vie conjugale d'anciens époux et établit des règles particulières au sujet de la dot et de la succession. L'article 308 vise, pour sa part, les communautés chrétiennes et juives, qui sont autorisées à suivre leurs propres règles en matière de fiançailles, 
de mariage, de divorce, de dot, de pension et de garde. Le Code de statut personnel constitue ainsi le droit commun de tous les Syriens auxquelles sont apportées de nombreuses exceptions qui sont fonction de la confession des individus.

L'amendement du Code de statut personnel de 1975 a introduit des changements importants sur les questions de polygamie, de dot, de pension d'allaitement, d'élévation de l'âge de la garde des enfants par leur mère, d'entretien des enfants et de divorce par consentement $\left(\mathrm{khul}^{ }\right)$.

S'agissant de l'âge de la garde des enfants, l'article 146 du Code de statut personnel, tel qu'amendé par l'article 19 de la Loi № 34 de l'année 1975, stipule que «la durée de la garde [par la mère] s'achève quand le garçon a atteint l'âge de neuf ans et la fille [celui] de onze [ans]». Précédemment, l'âge était fixé à 7 ans pour le garçon et 9 ans pour la fille. En 2003, la même disposition fut à nouveau amendée par la Loi $N^{\circ} 18$, votée par le Parlement le 19 octobre et promulguée par le Président le 25 octobre. L'article 146 du Code de statut personnel est désormais formulé de la manière suivante: «la durée de la garde [par la mère] s'achève quand le garçon a atteint l'âge de treize ans et la fille [celui] de quinze [ans]».

\section{L'ADOPTION DE LA LOI EN CONTEXTE ET EN ACTION}

Il s'agit à présent de décrire le cours complet du débat, avec ses différents intervenants, ses registres argumentatifs et son déploiement séquentiel. Nous entreprenons donc d'analyser le débat en contexte, celui-ci étant entendu comme l'ensemble des éléments de pertinence vers lesquels les participants s'orientent.

Le contexte de ce débat à l'intérieur de l'Assemblée du Peuple est constitué des éléments de pertinence d'un processus dialogique: l'échos à des prises de position extérieures à l'enceinte; la référence aux exigences institutionnelles et procédurales; l'alignement sur des considérations d'ordre politique et législatif; l'orientation vers des audiences, matérielles ou virtuelles. Tout au long du débat sur l'élévation de l'âge de garde des enfants, ces éléments de pertinence sont manifestés, explicités, utilisés par les membres de l'Assemblée à toutes fins législatives pratiques, c'est-à-dire dans l'accomplissement de leur activité de fabrique de la loi.

\section{Le déroulement du débat}

Le débat commence par le rappel, par le Président de l'Assemblée, de la procédure suivie: dépôt du projet de loi par le gouvernement, travail en Commission des Affaires constitutionnelles et législatives, soumission de la proposition amendée par la Commission à l'Assemblée du Peuple (Président, ligne 1) ${ }^{1}$. Lecture est ensuite faite par le greffier des textes du projet de loi présenté par le Gouvernement et de la proposition amendée par la Commission (greffier-8). La parole est ensuite donnée aux parlementaires ayant fait connaître leur désir de s'exprimer. Dans un premier temps, le débat se déroule normalement, avec des interventions favorables à la proposition de la Commission (orateurs 1/54 et 2/81)

1 Dans la suite du texte, le premier chiffre fait référence à l'ordre de passage de l'intervenant et le deuxième, à la ligne correspondante de sa prise de parole dans la retranscription complète. 
et d'autres favorables à une modification du texte (orateurs 3/149 et 5/205). A ce stade, une demande de verrouillage du débat (qifl bâb al-niqâsh), c'est-à-dire de confinement du débat et de mise au vote, survient (orateur 6/238).

Le verrouillage permet donc de clôturer un débat en passant à la discussion du projet article par article. Mise au vote, la proposition de verrouillage est adoptée à la majorité des membres présents. Une fois le verrouillage voté, le Président demande logiquement au greffier de procéder à la lecture du premier article (Président/245). Il s'ensuit une contestation du verrouillage (orateur 8/323) qui inaugure un deuxième temps du débat au cours duquel se succèdent une série de prises de position affichant sur toute la gamme des possibles: en faveur du projet gouvernemental (orateurs 13/391, 14/405, 17/480 et 20/566), en faveur de la Commission (orateur 9/327), en faveur d'une tierce solution (orateurs 15/413 et $16 / 466$ ) ou encore en faveur de la proposition déjà faite dans la première phase du débat (orateurs 18/499, 19/563 et 21/578). A ce stade, il semble bien que la discussion, débridée, soit polarisée entre les partisans du projet du gouvernement, ceux de la proposition de la Commission et ceux de la proposition d'amendement formulée par l'orateur 3 (proposition Habash). Une demande de verrouillage survient à nouveau, adoptée à la majorité (ligne 590), de même qu'un vote sur la proposition de la Commission, qui se trouve rejetée (ligne 596). La situation est confuse et le Président décide de suspendre de la séance.

A la reprise de séance, nous entrons dans une troisième phase du débat, marquée par des mises au point procédurales (Président/619), une série de votes sur les différentes propositions introduites par des membres de l'Assemblée. C'est ainsi que les propositions Nijâtî (643) et Shâhîn (652) sont rejetées, tandis que la proposition Habash (659) est adoptée. S'ensuit une succession de points d'ordre soulevés par des parlementaires et portant sur la procédure de vote, ce qui conduit le Président à procéder à un vote par assis et levés (ligne 683) confirmant l'adoption de la proposition Habash à une majorité de 87 voix ( 75 contre). Le Président ouvre à ce moment le débat sur l'ensemble de la loi (691), à quoi répondent des commentaires sur un problème de compétence des tribunaux (orateur 9/693) et de nouveaux points d'ordre relatifs au vote et au quorum de présence (orateurs 24/701, 5/707, 25/717, 24/727). La dernière intervention, elle aussi sur ce point d'ordre, préfigure la clôture du débat, en ce qu'elle souligne le caractère oiseux de la discussion (orateur 10/739). Le Président enchaîne par une mise au vote de l'ensemble de la proposition Habash, qui est adoptée à la majorité (ligne 744), déclare que «le projet est adopté et [...] devient loi » (Président/745) et, ayant de la sorte conclu le débat sur l'âge de la garde, passe au point suivant de l'ordre du jour en utilisant une formule de transition («A présent, [chers] collègues, [...]») (Président/759).

Cette présentation générale du déroulement du débat permet d'observer à quel point celui-ci est un accomplissement pratique contextuel et endogène. L'ouverture de la discussion, le rôle du président, la répartition des tours de parole, le recours au vote, la décision d'une suspension de séances, autant d'éléments propres à l'ordre interne du débat parlementaire en contexte et en action. Ainsi, loin d'être totalement prédéterminé et prévisible, le débat suit un cours ordonné, mais en même temps occasionnel, ponctuel, contextuel et proprement spécifique.

Les travaux portant sur les débats parlementaires sont peu nombreux. On observe toutefois une attention croissante accordée à leur organisation discursive. 


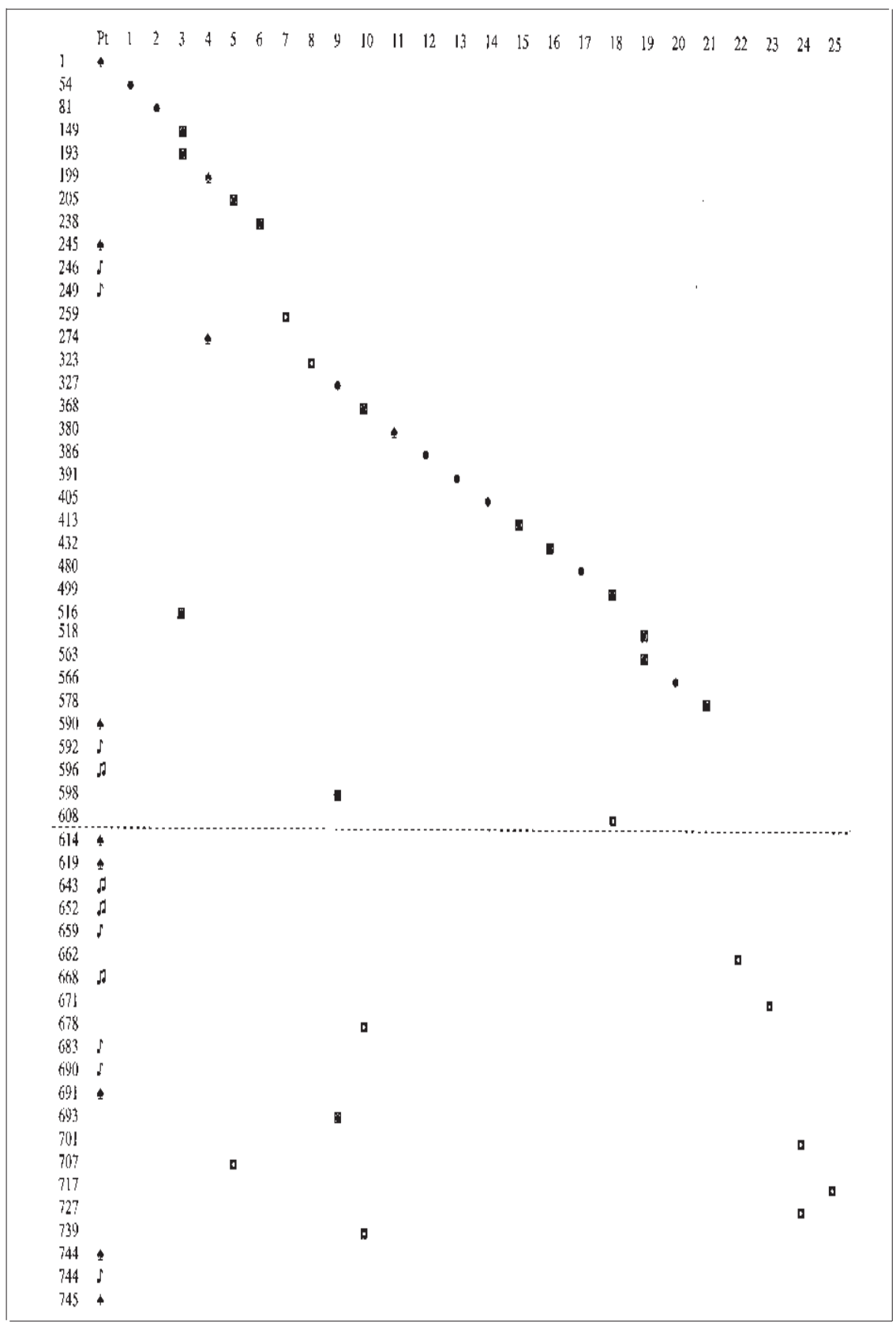

Lègende: : : tour de parole du Prévident de l'Assemblée : lecturc du grefficr; $\bullet$ : tour de parole favorable au projel du

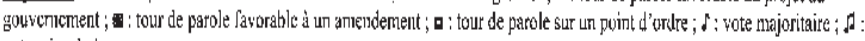
vote minotitaire 
Il en ressort que ce type de discours présente une spécificité marquée, bien que chacune de ses composantes, prise isolément, ne lui soit pas exclusive. Comme dans d'autres contextes institutionnels, la sélection des orateurs et le contrôle des tours de parole sont assurés par un président. On remarque également que le temps alloué à chaque tour de parole est limité, au moins implicitement. La manière de s'adresser au public de l'assemblée est particulière, de même que le lexique et la syntaxe des orateurs sont spécifiques, plus élaborés en tout cas que dans la conversation ordinaire. Plutôt cependant que d'établir une liste générale et abstraite des spécificités du débat parlementaire, nous proposons de le voir comme un processus endogène dont les particularités se déploient in situ. Autrement dit, c'est à partir du débat sur l'âge de la garde des enfants que les propriétés de la fabrique parlementaire de cette loi spécifique émergeront: «le dialogue parlementaire constitue un processus qui se déploie dans l' 'ici et maintenant', avec des résultats et des conséquences imprévisibles » (Ilie 2003: 35).

\section{Le pivot présidentiel}

Dès l'ouverture même du débat, le Président projette l'objet de la séance de manière explicite:

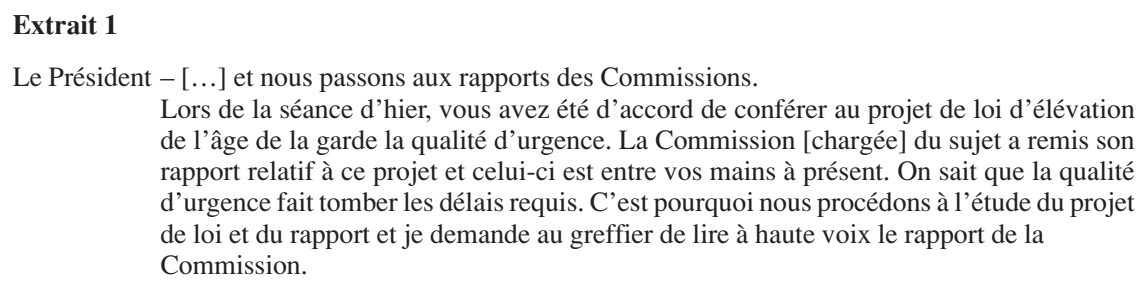
de l'âge de la garde la qualité d'urgence. La Commission [chargée] du sujet a remis son rapport relatif à ce projet et celui-ci est entre vos mains à présent. On sait que la qualité d'urgence fait tomber les délais requis. C'est pourquoi nous procédons à l'étude du projet de loi et du rapport et je demande au greffier de lire à haute voix le rapport de la Commission.

Pour assurer le passage entre l'objet de discussion précédent et le nouvel item, le Président utilise un verbe de transition («nous passons »). Ayant ainsi préfacé la transition, il procède à l'introduction du point à l'ordre du jour en rappelant les conditions procédurales justifiant qu'il soit présenté à ce moment précis à l'attention des parlementaires. Ce faisant, le Président, invoquant l'urgence, s'attache à justifier que les règles relatives aux délais d'introduction des projets et propositions de loi soient contournées. Le Président fait référence à la séance de la veille - inscrivant de la sorte ses propos dans la continuité historique des travaux de l'Assemblée - lors de laquelle cette urgence a été décidée. On observe ici comment une disposition spécifique du Règlement intérieur, l'article 132 en l'espèce, sert de guide à l'action du Parlement:

Art.132 - Si le pouvoir exécutif ou les membres ont présenté un projet ou une proposition qu'il faut examiner rapidement, l'Assemblée est autorisée à décider de l'urgence de son examen.

Cette disposition du Règlement intérieur n'agit pourtant pas sur le comportement des protagonistes de manière inéluctable (la règle n'étant alors qu'une question d'application mécanique par des acteurs agis et non agissant). Elle n'est qu'un moyen pour les acteurs de produire des effets ou d'en éviter d'autres. La 
règle apparaît donc comme un objet «accomptable» (descriptible et justifiable), c'est-à-dire un objet dont on peut rendre compte et dont l'usage fait l'objet de justifications.

La prise de parole initiale du Président spécifie aussi immédiatement l'objet des travaux de l'Assemblée. En l'espèce, il s'agit de l'action légiférante du Parlement. Il ne suffit en effet pas de situer le cadre général de l'activité observée; il faut encore préciser le type d'activité que l'on observe dans ce cadre. Le Parlement est le lieu d'activités multiples (discussion de politique générale, questionsréponses, activité législative, etc.) et cela trouve un écho dans l'organisation des débats. Comme le souligne Bayley (2004: 24), «les parlementaires sont très explicites quant à ce qui se fait au Parlement». Dans le cas qui nous concerne, ce qu'ils font, c'est légiférer. Les différentes prises de position qui vont se succéder s'inscrivent dans cette finalité exposée dès l'entame. Autrement dit, une donnée explicite du contexte est établie, qui permet de saisir les faits et dires subséquents comme posés à toutes fins législatives pratiques. Le cadre procédural est, en outre, fixé dans le même mouvement: proposition du gouvernement, travaux de la Commission, rapport de la Commission, lecture du rapport, étude en séance. On tient ici une première manifestation de ce que tous les éléments de pertinence sont enchâssés dans le cours d'action, manifestés et explicités par des protagonistes compétents (c'est-à-dire dotés des moyens leur permettant de faire sens de leur environnement et de ce qui s'y produit). Comme le souligne très bien Bevitori (2004: 94), les questions posées dans le cadre de ce type d'événement de parole véhiculent des significations et des attentes qui sont ancrées dans l'activité ellemême. En ce sens, il est permis de dire que l'action des membres du Parlement qui vont intervenir est d'emblée inscrite dans une démarche institutionnelle orientée vers la production d'une pertinence législative et d'une correction procédurale (Dupret 2006).

Ainsi posé, le débat commence à se dérouler d'une manière ordonnée, autour d'un Président exerçant un rôle pivotal de distributeur des tours de parole. Le système parlementaire d'allocation des tours de parole, par le décalage temporel de la dimension dialogique des débats qu'il opère, empêche ceux-ci de prendre un caractère de confrontation directe. Le débat parlementaire présente donc un aspect dialogique d'un type très formel et peu interactif. L'interaction se déroule en face-à-face, mais dans un contexte où la relation est contrainte par les règles de procédure, formelles ou implicites, propres à l'institution. Dans le cas syrien, le débat est fait d'une succession de prises de parole transitant par un ordonnateur central:

\section{Extrait 2}

\begin{tabular}{|c|c|}
\hline 385 Le Président & - La parole est à [notre] collègue Ramadân `Atiyya. \\
\hline 386 M. Ramadân `Atiyya & - Monsieur le Président - Chers collègues. \\
\hline 387 & Nous autres discutons de l'article et, après avoir entendu l'opinion des \\
\hline 388 & collègues, nous écoutons l’opinion du pouvoir exécutif. Merci. \\
\hline 389 Le Président & - Cher collègue, nous allons discuter de ce sujet et nous allons enregistrer \\
\hline & Nâsir `Abd al-`Azîz. \\
\hline
\end{tabular}


Notons que le Président est le seul protagoniste qui s'avère être en situation d'interrompre les orateurs et de poser des questions «à chaud». L'interruption fait partie des modes d'allocation des tours de parole (Bevitori 2004: 87), même si elle peut être en théorie proscrite. En Syrie, elle est non seulement interdite par le Règlement intérieur (Art.43-B), mais elle n'appartient pas non plus au registre des pratiques courantes. Le président est donc le seul protagoniste exerçant pareille action de contrôle et de pouvoir sur l'orateur (Bevitori 2004: 103):

\section{Extrait 3}

182 M. Muhammad Habash - [...] Je pense qu'il est utile que la durée de la garde du garçon soit de 13 ans et soit limitée pour la fille à 15 ans, et qu'en référer au juge revienne à l'homme qui est plus apte à cela, sachant que la loi a stipulé les échéances de la garde et qu'il est possible à l'homme de réduire la garde s'il existe des causes impératives. C'est pourquoi j'engage à ce que nous soyons à la recherche de la justice. La justice absolue, c'est quelque chose qui n'existe pas, sauf chez Dieu Tout-Puissant. Nous nous efforçons de la chercher. De nombreuses mères ne peuvent pas veiller sur un enfant plus de deux ans et de nombreux pères ne sont pas en mesure de veiller sur un enfant.

Le Président - interrompant - Cher collègue Muhammad, votre proposition est de supprimer la mention (le juge a le droit d'étendre... etc.) et que [l'article] soit adopté sans elle. Est-ce que ce n'est pas le résumé du propos?

M. Muhammad Habash - poursuivant - Pour que je sois clair, je demande que l'article soit adopté comme suit: (la durée de la garde s'achève quand le garçon a atteint l'âge de treize ans et la fille l'âge de quinze ans) et la suppression complète du paragraphe b. Je marque ma sympathie pour l'opinion de mon ami Monsieur `Abd al-`Azîz al-Shâmî avec qui je suis d'accord quant à cette option. Merci. - La parole est à [notre] collègue le président de la Commission.

Le rôle du Président de l'Assemblée s'avère donc fondamental. On remarquera que sa fonction ressort du seul fait qu'il reprend la parole à chaque fin de tour d'un orateur et qu'il invite l'orateur suivant à parler. Autrement dit, sa fonction n'est pas un postulat mais un élément explicite du contexte, un élément que l'on saisit par la seule force de sa conséquentialité procédurale: la suite du débat en découle (Schegloff 1987; Van Dijk 2005 : 22). Le Président organise le débat, il y prend une part active, il l'oriente dans le sens de ses préférences. Son pouvoir de contrôle est quasiment absolu. Cela apparaît tout d'abord dans la gestion du temps de parole, qu'il cherche systématiquement à contenir:

\section{Extrait 4}

133 Mme Hûdâ al-Humsî
[...]L'élévation de l'âge de la garde est à 15 ans pour la fille et à 13 ans pour le fils, après quoi ils ont atteint un degré de développement psychique prescrivant de leur laisser le libre choix de vivre chez leur mère ou chez leur père. C'est ce qu'a indiqué l'Envoyé - que Dieu prie sur lui et lui donne la paix - quand est venue à lui une femme et qu'elle a dit: ô Envoyé de Dieu: «Mon époux veut partir avec mon fils alors qu'un puits [qui] arrose ses raisins m'est profitable. L'Envoyé a dit au fils: c'est ton père et c'est ta mère, prends la main de celui des deux que tu veux. Il a alors pris la main de sa mère et elle s'est éloignée avec lui ». Merci.

Je suis extrêmement patient et je poursuivrai avec vous jusqu'à sept heures du matin. Vous disposez du temps et vous pouvez évaluer le temps. Les limites naturelles pour tout intervenant sont de 4 minutes au maximum et si 


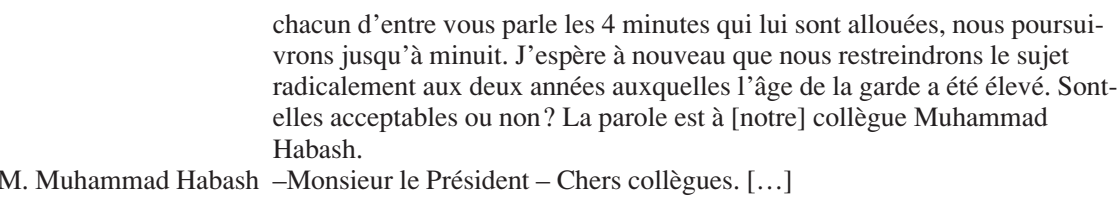

Il en ressort que le temps de parole est, lui aussi, une question accomptable, une question dont l'usage se trouve à la fois prescrit et justifié. En l'espèce, H. al-Humsî (orateur 2) développe un point de vue, long (lignes 81-141), que le Président ne commente pas sur le fond, mais qui sert d'appui à un tour de parole dans lequel il fait état de sa patience avec une certaine ironie (ligne 142: «Je suis extrêmement patient et je poursuivrai avec vous jusqu'à sept heures du matin»). C'est une force évidente de l'interruption et de l'ironie que de pouvoir faire «perdre la face» à celui ou celle qui en est la victime.

Par ailleurs, le Président saisit l'occasion de cette auto-désignation comme orateur pour préciser certaines règles de conduite relative à la gestion du temps (4 minutes, évaluation individuelle, nécessité de s'en tenir au sujet). Notons que ces règles ressortissent à un principe de «normalité» et de «moralité», mais procèdent de règles non conventionnelles (Bevitori 2004: 102). Le Président ne renvoie pas à des dispositions écrites (le Règlement intérieur de l'Assemblée, par exemple), mais à des « limites naturelles » (ligne 143), à une arithmétique élémentaire (lignes 144-145) et à la capacité de chacun à l'autodiscipline (lignes 145146). Ceci ressort tout aussi clairement de l'extrait suivant:

\section{Extrait 5}
- Merci, cher collègue, pour cet éclaircissement. Nous reprenons en prenant en considération l'opinion du président de la Commission et en espérant poursuivre la discussion dans ses limites évidentes, pour en arriver à un résultat sans entrer dans des [considérations] futiles. La parole est à [notre] collègue Ghâlib `Anîz.
- Monsieur le Président - Mesdames et Messieurs, chers collègues [...]

Le contrôle exercé par le Président apparaît également dans la régulation procédurale qu'il impose. A chaque fois que l'occasion lui en est offerte, il s'efforce en effet de favoriser le verrouillage du débat:

\section{Extrait 6}

237 Le Président 238 M. Ahmad Ghuzayl
- La parole est à [notre] collègue Ahmad Ghuzayl.

- Monsieur le Président - Chers membres [du Parlement] (al-sâda al$\left.a^{\prime} d \hat{a}^{\prime}\right)$.

J'ai présenté une proposition écrite au bureau de la présidence, sur la base de l'article 42 du Règlement intérieur, pour que l'on verrouille le débat et que l'on procède à la discussion des articles du projet de loi. Je souligne que toutes les propositions que les collègues ont présentées sont des propositions de valeur, mais elles tournent toutes autour de l'amendement de tel ou tel article. Je considère qu'il serait plus approprié de procéder à la discussion du projet de loi article par article. Merci.

- Ceux qui sont d'accord avec la proposition de [notre] collègue Ahmad Ghuzayl le montrent en levant la main / les mains ont été levées / majorité, accepté, nous verrouillons le débat. 


\begin{abstract}
A présent, ceux qui sont d'accord pour que l'on procède à la discussion du projet de loi article par article le montrent en levant la main / les mains ont été levées / majorité, Monsieur le greffier lise à haute voix l'article premier. Article 1 - L'article 19 de la loi no. 34 en date du 31/12/1975 est amendé et est adopté comme suit:

A - la durée de la garde s'achève quand le garçon a atteint l'âge de onze ans et la fille l'âge de treize ans.

$\mathrm{B}$ - le juge a le droit d'étendre la durée de la garde à treize ans pour le garçon et à quinze ans pour la fille s'il lui paraît que l'intérêt de l'enfant gardé l'impose.

- Vous avez entendu l'article. Quelqu'un a-t-il une observation [à faire] à son [sujet]? Il y a un point d'ordre. La parole est à [notre] collègue Muhî alDîn Habbûsh.
\end{abstract}

M. Muhî al-Dîn Habbûsh-Monsieur le Président - Chers collègues. [...]

Ce n'est pas tant une opposition aux différents amendements qui ressort de l'attitude du Président qu'un souci de procéder à la gestion expéditive de la discussion du projet. Quand un tour de parole lui semble peu explicite, il tend souvent à interrompre l'orateur pour ramener son intervention à une conception restrictive de l'objet du débat:

\title{
Extrait 7
}

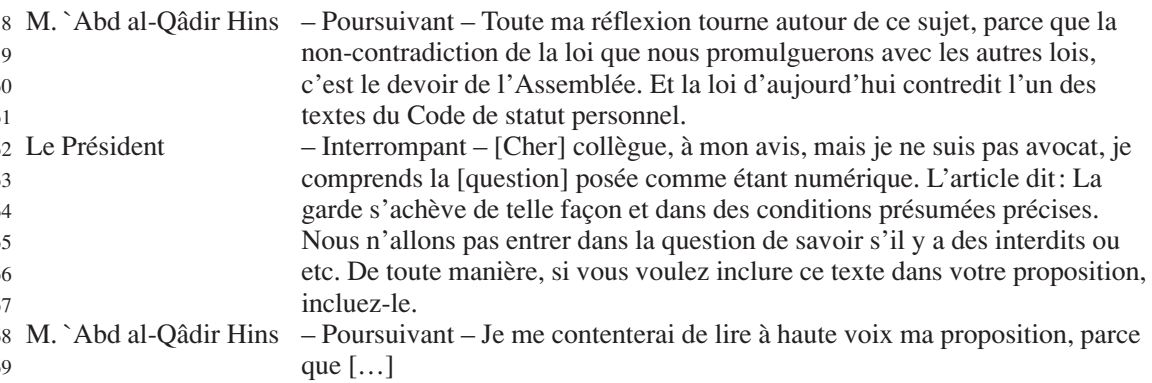

Le Président peut également intervenir pour poser à l'orateur une question de type oui-non. Ce type de questions vise de manière générale à initier un échange en requérant une information de l'orateur (Eggins 1997: 85). En polarisant les positions, elles exercent une fonction clarificatrice, en réduisant le caractère incertain du discours de l'orateur, et canalisent le débat dans la direction pratique du vote sur les projets et propositions:

\section{Extrait 8}

513 Le Président

514

515

516 M. 'Ammâr Bakdâsh
- [Cher] collègue, ce que j'ai compris de votre [intervention], c'est que vous refusez l'amendement et refusez le texte original et que vous êtes pour la proposition de [notre] collègue Muhammad Habash.

- Oui, c'est mon opinion. Merci.

Le contrôle du Président s'exerce aussi par sa capacité à rythmer le débat. Ainsi, quand celui-ci s'avère débridé, il décide d'une suspension de séance (tableau 1, lignes 590-608). En outre, il multiplie, sous des dehors pédagogiques, les rappels de procédure. Ce faisant, il ne manque pas de restreindre la capacité 
d'intervention effective des membres de l'Assemblée et de ramener les débats à leur seule finalité pratique, à savoir le vote sur le projet de loi :

\section{Extrait 9}

368 M. 'A.A. al-Mawsallî
369
370
371
372
373
374
375
376
377 Le Président
378
379
380
381
382
383 M. Nizâr al-`Asasî
384
- Monsieur le Président - Chers collègues.
Il est vrai que l'article 42 du Règlement intérieur permet à chaque membre de demander le verrouillage du débat et il revient à l'Assemblée à sa majo- rité d'en décider ainsi, mais le verrouillage du débat est une chose et l'audi- tion du pouvoir exécutif une autre chose. [Nous faisons] comme si le ministre de la Justice n'était pas présent et nous le considérons comme un membre et n'entendons pas son avis avant le procéder à la discussion des articles. [Notre] collègue Muhammad a proposé l'amendement de l'article, quel est l'avis du pouvoir exécutif à ce propos? Merci.
- Cher collègue, si nous entendons le ministre, est-ce que nous changerons la procédure? Dès lors que la question est controversée, il n'y a pas d'obs- tacle à entendre Monsieur le ministre et, ensuite, nous en reviendrons à soumettre l'article à la discussion. La parole est à Monsieur le ministre de la Justice
- Monsieur le Président - Chers collègues.
Ce sujet a été examiné en long et en large devant la Commission constitu- tionnelle $[\ldots]$

Le contrôle du Président opère également dans le sens d'une orientation des débats. Bien qu'on ait affirmé plus haut qu'il ne s'attachait pas à manifester explicitement ses préférences, il ressort de l'étude attentive de ses interventions que le Président œuvre dans un sens conforme au rapport de la Commission. Cela n'apparaît pas tellement comme l'expression d'une préférence de fond que comme la traduction d'un tropisme institutionnaliste le faisant pencher vers le respect des instances autorisées: représentants du gouvernement, président et rapporteur de la Commission des affaires constitutionnelles et législatives, etc. Il est vrai que le Règlement intérieur de l'Assemblée du Peuple autorise ces instances à intervenir dans le débat autant de fois qu'elles le considèrent utile, alors que les interventions des membres du Parlement sont limitées à trois (Art.43-C). Il n'en est pas moins manifeste, à bien considérer différentes interventions du Président, qu'il a une préférence marquée pour l'accomplissement formel de la procédure prévue pour la discussion des projets de loi. Dès lors, les interventions venant perturber cet accomplissement sont traitées comme des incongruités ou, du moins, comme des atteintes à la normalité procédurale qu'il escompte. L'extrait suivant en est l'illustration, parmi bien d'autres:

\section{Extrait 10}

M. Muhî
al-Dîn Habbûsh
Le Président

\footnotetext{
- Monsieur le Président - Chers collègues.

Nous sommes pour la démocratie, en dépit du [fait] que nous avons verrouillé le débat sur un sujet important qui a des répercussions sur la société tout entière. Je voudrais préciser que [notre] collègue le docteur Muhammad Habash a présenté une proposition écrite. La proposition écrite doit être lue à haute voix pour que nous votions dessus. Nous demandons au bureau de la présidence de la lire pour que nous l'entendions bien. Merci - Cher collègue Muhî al-Dîn, la proposition de verrouillage du débat a été mise au vote et a été adoptée à la majorité. Ensuite, après lecture à haute voix du projet article par article, nous présentons les propositions des
} 


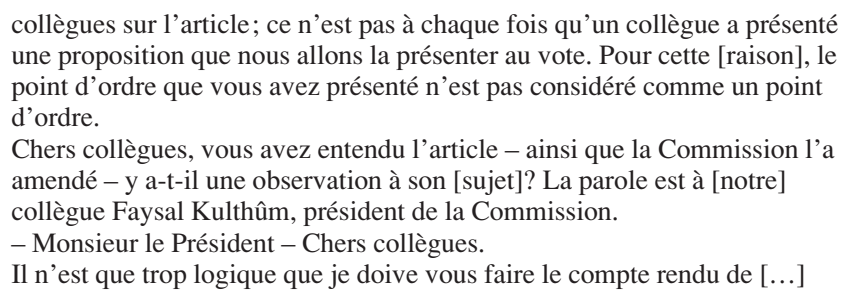
une proposition que nous allons la présenter au vote. Pour cette [raison], le point d'ordre que vous avez présenté n'est pas considéré comme un point d'ordre.

Chers collègues, vous avez entendu l'article - ainsi que la Commission l'a amendé - y a-t-il une observation à son [sujet]? La parole est à [notre] collègue Faysal Kulthûm, président de la Commission.

- Monsieur le Président - Chers collègues.

Il n'est que trop logique que je doive vous faire le compte rendu de [...]

Le travail d'orientation du débat accompli par le Président est manifeste dans sa gestion des tours de parole (interruptions, rappels de points d'ordre) aussi bien que dans ses prises de position, qui visent à restreindre l'étendue de la discussion et à faire valoir son orientation personnelle:

\section{Extrait 11}

- interrompant - Avec votre permission, [cher] collègue, le texte dit: L'article 19 de la loi no. 24 du 21/12/1975 est amendé. Dès lors, c'est inclus dans les présomptions, et nous ne sommes pas en train de réexaminer toutes les lois. Quand donc le législateur l'a établi, il en comprenait parfaitement les fondements. Maintenant, le sujet, en toute simplicité, avec l'évolution du monde et le désir du gouvernement de changer l'âge de 11 à 13, il s'est présenté avec ce projet. Pour que nous en restions à ce sujet et pour que nous ne rentrions pas dans les attendus, nous sommes à présent concentrés sur un point et je le considère comme [un point] numérique, contrairement à ce que vous avez affirmé, [cher] collègue.

\section{Les tours de parole des membres de l'Assemblée}

La prise de parole des orateurs débute systématiquement par une forme d'adresse conventionnelle ( «Monsieur le Président - Chers collègues (al-sâda alzumalâ’)») qui connaît quelques rares variantes: «Monsieur le Président Mesdames et Messieurs, chers collègues (al-zamîlât wa'l-zumalâ')» (ligne 205); «Monsieur le Président - Chers membres [du Parlement] (al-sâda al-a ¿ầ')» (ligne 238). Il faut bien constater qu'entre autres choses, «le style de l'intervention indexe la formalité de la rencontre en général, et la formalité de l'interaction parlementaire en particulier»(Van Dijk 2005: 25).

La forme prise par le tour de parole est par ailleurs étroitement tributaire du moment du débat auquel elle se situe. C'est ainsi que les tours correspondant à ce que nous avons appelé la troisième phase du débat (après la suspension de séance) sont entièrement configurés par la procédure de vote engagée à ce moment. En revanche, les tours correspondant à la première phase du débat, jusqu'au premier verrouillage (ligne 247), consistent en des prises de position indépendantes les unes des autres, manifestement préparées à l'avance, dans une relation dialogique avec le projet de loi mais pas entre eux. Ces tours, longs, argumentés et peu interactifs, polarisent le débat et positionnent les protagonistes. Ils sont généralement construits de manière rhétorique, en trois parties (introduction, motivation, conclusion): 


\section{Extrait 12}

4 Mme Wa'd Khaddâm - Monsieur le Président - Chers collègues (al-sâda al-zumalâ’).

La garde est un droit de l'enfant et elle a été légiférée dans son intérêt. Tel est le principe fondamental qu'ont établi le fiqh et la loi. L'interprétation se fait dans l'intérêt de [l'enfant] gardé dans tous les domaines. Du fait que la question de l'éducation des enfants diffère dans les sociétés modernes de ce qu'elle [était] dans les sociétés anciennes et impose le changement de l'infrastructure de ces sociétés, on demande d'allonger la durée de la garde. Tout comme l'application de la loi sur l'enseignement fondamental obligatoire jusqu'à la fin du cycle préparatoire, on demande l'allongement de la durée de la garde en sorte que l'[enfant] gardé n'ait pas à changer le lieu de résidence et de vie auquel il est habitué et, en particulier, l'école et l'environnement scolaire.

A la base, les opinions des jurisconsultes et des législateurs ont différé sur la durée de la garde en fonction des différences de situation sociale et des différences dans les besoins éducatifs des enfants.

C'est pour cela que nous considérons que l'amendement proposé protège cet intérêt et je demande que soit [donnée] au juge l'appréciation du meilleur intérêt de l'[enfant] gardé dans chaque affaire ('alâ hidda???). Avec l'augmentation de la pension alimentaire (nafaqa) destinée à chaque enfant et la garantie du logement de l'[enfant] gardé, ce qui est une question importante qu'on ne peut pas supprimer ou absorber dans son règlement financier, dans la mesure où la question du logement est devenue difficile et exorbitante et où la gardienne ne peut plus la garantir dans la plupart des cas, en particulier si la mère a plus d'un enfant, si la maison de sa famille est petite et si elle ne travaille pas. Cette question a été résolue en Tunisie, Algérie, Egypte, Irak, Yémen, Koweït.

Pour tout ceci, je suis en faveur du projet de loi et de la vitesse de sa promulgation. Merci.
\end{abstract}

Dans la deuxième phase du débat, les tours de parole prennent un aspect plus circonscrit et consistent à prendre position en faveur de l'un ou l'autre des textes présentés ou à argumenter sur des points d'ordre et, en particulier, sur des questions de vote. Cela s'explique par le fait que, le débat ayant été verrouillé, c'est la discussion du projet article par article qui est engagée. Pourtant, le débat ne change pas radicalement d'objet. Peut-être, tout d'abord, parce qu'il s'agit d'un projet de loi portant sur un article unique, ce qui réduit fortement la distinction entre discussion générale et discussion sur les articles séparément. Cela tient sans doute aussi au fait que le verrouillage n'a pas atteint le résultat escompté en ce sens que, d'une part, sa légitimité est remise en cause (orateur 7/259-264 et 8/323325 ) et, de l'autre, de nombreuses positions et propositions d'amendement restent à présenter. Cette deuxième partie du débat se présente donc comme une succession de mouvements d'alignement sur les différents pôles: projet du gouvernement, proposition de la Commission, propositions des différents parlementaires (cf. annexe 3, lignes 274-589). Après avoir été défendue par le Président et le rapporteur de la Commission (orateurs 4/274-321 et 9/327-366), la proposition de cette dernière essuye un tir de barrage (orateurs 13/391-403, 14/405-411, 17/480497 et 20/566-576), tandis qu'on observe une montée en puissance de la proposition Habash, indépendamment de toute intervention de celui-ci(orateurs 18/499516, 19/518-564 et 21/578-589). Le débat se cristallise autour de deux questions: l'âge de l'élévation et le pouvoir d'appréciation du juge. Elles se trouvent progressivement mises en équation (orateur 15/413-430), de telle sorte que la proposition 
visant à augmenter l'âge, au-delà du projet du gouvernement, tout en n'attribuant pas de pouvoir d'appréciation au juge, contrairement à la proposition de la Commission, finit par s'imposer comme une figure de compromis. Notons, à cet égard, que le compromis n'est pas le résultat d'une négociation substantielle, mais l'aboutissement d'un processus au cours duquel se sont juxtaposées des prises de parole contrastives les unes par rapport aux autres (allomorphes) ou alignées les unes sur les autres (isomorphes). La seconde demande de verrouillage intervient à ce moment. Une fois adoptée, l'Assemblée vote sur la proposition de la Commission. Le rejet de cette dernière, parce qu'il remet en cause la décision d'une instance majeure du Parlement et traduit l'émergence d'une majorité favorable à la proposition Habash, justifie une interruption de séance certainement destinée à clarifier la situation, à préciser la procédure à suivre dans ce cas de figure inhabituel et à prendre la mesure de la position majoritaire.

\section{Les audiences pertinentes}

Dans leurs tours de parole respectifs, les orateurs s'adressent à une audience. Celle-ci peut être matérielle (les personnes présentes dans l'enceinte) ou virtuelle (le public au sens large). L'analyse de discours s'est efforcée de dresser une typologie des participants aux débats parlementaires. Dans cette perspective, une première distinction est faite entre participants et non-participants. Dans la catégorie «participants», l'on retrouve l'orateur, les destinataires directs de son discours et tous ceux qui prennent part au débat mais ne sont pas les destinataires immédiats du tour de parole en cause. Les autres personnes tombent dans la catégorie des «non-participants». Ce sont les personnes ouvertement co-présentes mais extérieures au débat (des auxiliaires, bystanders) ou celles qui écoutent sans que l'orateur n'en soit conscient (des oreilles indiscrètes, eavesdroppers), avec de nombreux cas intermédiaires (Ilie 2003: 46). Cette typologie, pour cohérente qu'elle puisse paraître, n'en pose pas moins un certain nombre de problèmes, dès lors qu'elle ne procède pas de la manifestation endogène du statut de chacun, mais de l'imputation d'une qualité par un observateur extérieur. Elle présume de l'activité de chacun indépendamment de toute attestation empirique, dans un mélange de respect des catégories formelles (les membres du Parlement, les greffiers et secrétaires, le public de la galerie) et de prise en compte d'éléments de sens commun (ceux qui entendent sans être vus, les oreilles indiscrètes). Pourtant, la qualité de chacun est une propriété émergente, un statut qui se revendique, s'attribue et fait l'objet d'orientations expresses dans le cours même du débat, indépendamment de toute catégorie préétablie. Ainsi, l'orateur fait-il l'objet d'une allocation explicite d'un tour de parole par le Président («La parole est à [notre] collègue...»), tandis que le Président atteste de son statut par l'ensemble des actions décrites précédemment, au travers desquelles il s'auto-attribue sa qualité de Président, mais se voit également reconnaître cette qualité, publiquement, par le respect manifesté à sa position de distributeur des tours de parole et par les termes d'adresse utilisés par les orateurs («Monsieur le Président - Chers collègues »). Ainsi en va-t-il aussi du greffier, auquel il est explicitement demandé d'intervenir, ès fonction, et qui manifeste publiquement son statut par l'enchaînement sur l'action demandée auquel il consent: 


\section{Extrait 13}

5 Le Président -

[...] fait tomber les délais requis. C'est pourquoi nous procédons à l'étude du projet de loi et du rapport et je demande au greffier de lire à haute voix le rapport de la Commission.

Monsieur le Président de l'Assemblée du Peuple

La Commission des affaires constitutionnelles et législatives a tenu une séance à onze

Ce sont donc les orientations explicites de tous les participants à l'interaction qui font émerger les statuts, fonctions et rôles de chacun, de même qu'elles constituent les audiences auxquelles ils s'adressent. L'audience n'est dès lors plus faite de l'ensemble des gens qui écoutent et participent du point de vue surplombant et putatif d'un chercheur omniscient, mais des objets vers lesquels s'orientent les personnes engagées dans ce cours d'action (Assemblée, Président de l'Assemblée, Collègues, Commission, président de la Commission, membre de l'Assemblée nommément désigné, etc.) et/ou des objets revendiquant ce statut dans ce même cours (Président de l'Assemblée, membre de l'Assemblée en son nom propre, président de la Commission ès qualité, rapporteur de la Commission ès qualité, etc.). Tour à tour, les multiples audiences se manifestent ou sont désignées, en ce compris un public virtuel (absent de l'enceinte), qu'il s'agisse de celui auquel la télévision et la presse rendent accessibles les débats ou de l'instance (électorat, autorités politiques, autorités partisanes) à laquelle l'élu est redevable de son élection et donc comptable de son activité parlementaire. Dans le débat que nous étudions, cette instance politique n'apparaît pas, contrairement à un autre débat de l'Assemblée du Peuple portant sur la création d'un Organisme syrien pour les affaires de la famille, au cours duquel référence est fréquemment faite au Président de la République, comme l'atteste l'extrait repris ci-dessous:

\section{Extrait 14}

$\begin{array}{ll}99 \text { M. Muh. Kh. Najâtî } & \text { - La conférence nationale «La femme et l'éducation à Damas » s'est tenue à } \\ & \text { Damas sous la supervision éclairée (ri âya ‘aqliyya) de Monsieur le Prési- } \\ \text { dent. Tout ceci s'inscrit sur une trajectoire de modernisation et de dévelop- } \\ \text { pement que conduit son excellence le Président de la République, le Prési- } \\ \text { dent Bashshâr al-Asad, qui est dans le prolongement dans ce domaine de la } \\ \text { voie du leader éternel Hâfiz al-Asad, qui a dit: La famille stable est la pierre } \\ \text { angulaire de la construction sociale. }\end{array}$

L'orientation vers une audience virtuelle, bien qu'elle ne soit pas fréquente, revêt deux formes possibles, en règle générale. Il s'agit soit d'une invocation de la «société», senso latu:

\section{Extrait 15}

- Monsieur le Président - Chers collègues.

Il n'est que trop logique que je doive vous faire le compte rendu de ce qu'il y a eu comme débat au sein de cette Commission qui était ce jour-là assaillie par les membres [de l'Assemblée]. La question n'a pas été restreinte aux membres de la Commission des affaires constitutionnelles et législatives, mais la pièce était pleine de membres de l'Assemblée et j'ai donné la liberté à l'ensemble des membres, qu'ils soient membres originaires de la Commission ou qu'ils soient extérieurs à la Commission. 


\begin{abstract}
Le débat fut manifestement libre. Nous en sommes arrivés à la [conclusion] que le projet présenté par le gouvernement n'était pas compatible avec ce qui est requis dans la vie sociale et la vie familiale, en dépit du fait que le projet du gouvernement est apparu, dans la discussion générale, clairement compatible avec les autres lois pour ce qui a trait à la responsabilité pénale du fils. Nous avons également un apport à ce débat. Le droit [islamique] [...]
\end{abstract}

Il peut sinon s'agir d'une invocation de la «démocratie» (sous-entendant dès lors la prééminence de la majorité et le respect des opinions minoritaires):

\title{
Extrait 16
}

M. Muhî al-Dîn Habbûsh- Monsieur le Président - Chers collègues.

Nous sommes pour la démocratie, en dépit du [fait] que nous avons verrouillé le débat sur un sujet important qui a des répercussions sur la société tout entière. Je voudrais [...]

\section{Registres argumentatifs, catégorisations et pertinences}

Indépendamment ou en liaison étroite avec l'orientation vers ces audiences, les orateurs mobilisent des registres argumentatifs et catégoriels multiples. On peut, pour aborder cette question, reprendre la distinction entre argumentation (formulation d'assertions prétendant à la validité) et négociation (formulation de menaces et de promesses prétendant à la crédibilité) et la notion qui s'en dégage d'un usage stratégique de l'argumentation, c'est-à-dire de l'énonciation sous forme argumentative d'enjeux propres à une négociation et à des intérêts personnels ou partisans (Elster 2005: 59). Dans le contexte de ce débat, les parlementaires agissent donc, face à une audience multiple et en fonction des règles parlementaires, à toutes fins pratiques, par quoi on entend qu'ils «performent» leurs qualités d'élu, de législateur, de personne chargée de débattre de la chose publique, de politicien, mais aussi d'individu agissant à titre personnel.

Il nous importe dès lors d'analyser la forme que prennent les énoncés des différents orateurs de la première partie du débat et d'observer comment les registres argumentatifs et les stratégies discursives trouvent à s'entremêler. Quand elle prend la parole (orateur 1/54-79; cf. extrait 12), Wa'd Khaddâm apparaît explicitement comme une femme. Ceci se manifeste aussi bien physiquement que par l'indexation générique opérée par la mention du prénom («Wa'd») par le Président de l'Assemblée, quand il lui donne la parole, et se traduit dans la retranscription officielle par l'utilisation de la civilité devant le nom («Mme»). Cette catégorisation pave la voie, logiquement bien que pas nécessairement, du recours à un dispositif catégoriel organisé autour de l'enfant. Celui-ci est présenté comme doté d'intérêts et de droits (ses besoins en matière d'éducation, d'enseignement, de moyens de subsistance, de garde) qu'il convient de préserver. C'est au droit, à la fois musulman et moderne, qu'il revient d'œuvrer à cette fin. Le raisonnement de l'orateur procède donc du syllogisme suivant: (a) l'intérêt de l'enfant est d'être gardé en fonction de ses besoins spécifiques; (b) dans une société moderne, l'enfant a besoin d'être gardé par sa mère plus longtemps; (c) le droit syrien veille à l'intérêt de l'enfant dans la société moderne en prolongeant la durée de sa garde par la mère. Le pivot de ce syllogisme réside dans la notion de modernité. Le 
raisonnement procède ainsi sur la base d'un implicite en fonction duquel l'éducation dans une société moderne imposerait de confier l'enfant plus longtemps à sa mère. Autrement dit, le dispositif catégoriel que nous voyons à l'œuvre est celui qui unit l'enfant à sa mère pour les besoins d'une éducation dont la durée augmente avec la modernité de la société. C'est exactement le même raisonnement qui est suivi par Ghâlib 'Anîz (orateur 5), à ceci près (mais la différence est évidemment fondamentale dans ses implications) que c'est le père qui est présenté comme plus à même de former l'enfant aux difficultés de la vie moderne:

\title{
Extrait 17
}

205 M. Ghâlib `Anîz

\begin{abstract}
- Monsieur le Président - Mesdames et Messieurs, chers collègues (alzamîlât wa' l-zumalâ').

Dieu a gratifié la femme de qualités et de mérites énormes et Il l'a élevée à une place éminente dans la société. La réussite et le succès n'arrivent qu'avec l'accord des deux parents, la mère et le père.

La femme constitue la moitié de la société et l'homme l'autre moitié. On dit dans le proverbe populaire: (l'homme est... et la femme...). Qui pourrait priver la femme de son droit? Elle est l'artisane des hommes, elle est la mère, la sœur et l'épouse et, sans elle, ils ne prospèrent pas. Le Prophète que Dieu le bénisse et lui donne la paix - dit: «Les femmes sont les compagnes des hommes ». La femme, c'est celle qui est tendre et affectueuse, elle est la base et le fondement du succès des familles, de leur développement et de leur bien-être.

Le bonheur de la femme se reflète positivement sur le bonheur de la famille. Mais cette affection ardente et cette tendresse énorme la rendent incapable je ne dis pas toujours mais le plus souvent - de contrôler les jeunes garçons quand ils deviennent adolescents, de même que les filles quand elles atteignent un âge plus avancé.

La garde, cela ressort de son nom même, concerne un âge précoce et non un âge plus avancé. Il y a des femmes qui portent le bien en elle, tout comme des hommes, et l'inverse aussi.

Peut-être que l'âge auquel ce projet est arrivé, après une étude extensive, est le plus adéquat et le plus proche de la raison, de chaque opinion, de sa direction et de son but. Je suis pour l'amendement de la Commission, mais [je souhaite] que cette extension soit telle qu'il n'y ait pas lieu au différend judiciaire et à l'antagonisme, que cette extension que la Commission a proposée se fasse à la demande et dans la chambre de délibération, en sorte qu'aucun des deux époux ne soit contraint à présenter une requête et à la poursuivre en longueur, que s'exprime l'opinion d'un comité de deux arbitres, comme c'est le cas en matière de séparation (tafrîq) (un arbitre de sa famille à lui et un arbitre de sa famille à elle) et d'une personne distincte (mumayyiz) que les deux arbitres choisissent, connu sous le nom de comité d'arbitres (hay' a muhakkamîn), un arbitre de sa famille à lui, un arbitre de sa famille à elle et une personne distincte que choisissent les deux arbitres. Peut-être le bien viendra-t-il de cela, Dieu est le Conciliateur. L'instruction n'a pas de rapport avec ce sujet. Merci.
\end{abstract}

Nous voyons dès lors comment le débat parlementaire fonctionne sur le mode de la solidarité sans consensus: solidarité sur les termes fondamentaux (modernité, droit, intérêt de l'enfant); dissensus sur les conséquences à en tirer (droits de la mère vs droits du père). C'est, en quelque sorte, à une compétition sur la maîtrise de la définition des éléments propres à un dispositif catégoriel que nous assistons. Le dispositif en question est celui de la famille, avec sa collection d'élé- 
ments (les parents, les enfants), dont certains sont appariés (père-mère, enfantsparents, frères/sœurs-frères/sœurs). Des activités sont liées aux catégories de ce dispositif: se marier, divorcer, vivre ensemble, éduquer, garder, entretenir, etc. $\mathrm{Ce}$ sont les relations entre les différents éléments appariés et les activités qui sont liées au dispositif et à ses éléments qui constituent l'enjeu de ce conflit, non pas de catégorisation, mais d' «apportionnement» des droits et devoirs liés aux différents éléments du dispositif. L'on voit ici comment les catégories de sens commun trouvent à se déployer de manière clivée, sur la base des mêmes composantes et des mêmes jeux de rattachement, en prétendant toujours à la normalité/naturalité des configurations relationnelles qu'elles établissent. L'on peut de la sorte observer l'existence de deux types symétriques de conflits: d'une part, les conflits de catégorisation (par exemple, la qualification de «papa», de «père» ou de «chef de famille»); de l'autre, les conflits d'apportionnement, où ce n'est plus l'appartenance à une catégorie qui pose problème mais la détermination des droits et devoirs normaux/naturels qui s'y rattachent (le père est « responsable», la mère est «tendre», etc.).

Après Wa'd Khaddâm, c'est au tour de Hûdâ al-Humsî de prendre la parole. Ici aussi, la civilité de l'orateur est établie aussi bien physiquement que par l'indexation générique opérée par la mention du prénom. De la même façon, cette catégorisation préalable semble justifier la prise de parole en tant que membre de la catégorie «femme» et, à ce titre, du dispositif catégoriel de la «famille»:

\section{Extrait 18}

81 Mme Hûdâ al-Humsî Monsieur le Président - Chers collègues.

\author{
Au nom de Dieu le Clément le Miséricordieux \\ «Notre Seigneur, donne-nous, en nos épouses et nos descendants la joie des \\ yeux et fais de nous un guide pour les pieux $»^{2}$ \\ Vérité du Dieu Majestueux \\ La Loi islamique (sharî‘a islâmiyya) indulgente confirme que la femme est \\ un être humain doué de dignité et d'une personnalité juridique \\ indépendante. Elle a pratiqué, depuis l'émergence de l'islam, c'est-à-dire \\ depuis plus de mille quatre cents ans, la politique, l'allégeance et le combat \\ sur la voie de Dieu. Elle n'a jamais été éloignée des affaires de la société et \\ de la vie publique \\ Et Dieu - gloire à Lui le Très-Haut!, quand Il a créé l'être et a créé un mode \\ de vie et de subsistance complémentaire, a regardé les gens avec \\ équanimité, qu'il soient hommes ou femmes, et Il n'a pas distingué entre \\ eux. Tous deux se complètent dans cette vie et chacun a sa place. \\ Je demande protection à Dieu contre Satan le Lapidé \\ Au nom de Dieu le Clément le Miséricordieux \\ «Et celui qui fait de bonnes œuvres, homme ou femme, tout en étant \\ croyant, ceux-là rentreront au Paradis et ne subiront pas l'injustice, fût-ce \\ d'un creux de noyau $»^{3}$ \\ Vérité du Dieu Majestueux \\ (sourate des Femmes, verset 124) \\ L'Envoyé de Dieu - que Dieu le bénisse et lui donne la paix - a particulière- \\ ment mentionné le dévouement de la mère, si bien qu'il a ordonné à celui \\ qui lui posait la question: ô Envoyé de Dieu, quelle est la personne la plus \\ digne de mon compagnonnage vertueux? Il a dit: ta mère; il a dit: qui
}



ensuite? Il a dit: ton père.

Et parmi les propos de notre seigneur Jésus - sur lui soit la paix - dans la sourate de Marie:

Je demande protection à Dieu contre Satan le Lapidé

Au nom de Dieu le Clément le Miséricordieux

«Et la bonté envers ma mère, et Il ne m'a fait ni violent ni malheureux » ${ }^{4}$ (sourate de Marie, verset 32)

Il est rapporté par un des Compagnons que chaque fois qu'il s'en allait de chez sa mère, il la saluait et disait:

«La paix soit sur toi ô mère, et la clémence de Dieu et Ses bénédictions, Dieu te fut Clément de la façon dont tu m'as élevé [quand j'étais] petit; et elle lui répondait en disant:

sur toi soit la paix et la clémence de Dieu et Ses bénédictions: Dieu te fut Clément de la façon dont tu m'as aimée filialement [quand tu étais] grand ». (al-Adab al-mufrad de Bukhârî)

Dans les Lois célestes, est révélé ce qui est pour le bien de l'être humain et pour la réalisation de la justice dans la société. Elles sont fondées sur ce qu'il y a de bon dans les être humains. Les être humains sont toutefois faillibles et vulnérables, c'est pour cela qu'il faut nécessairement que soit promulgué un texte organisateur qui règle les relations des gens entre eux, et cela aussi à l'intérieur d'une seule et même famille.

Le projet de loi d'élévation de l'âge de la garde n'est pas une demande féminine. C'est une aspiration de la femme aussi bien que de l'homme. Les femmes sont les compagnes des hommes. L'homme, c'est le fils, le frère, l'époux et le père. Nous autres, par la grâce de Dieu, nous jouissons dans cette région de liens familiaux importants qui font de nous une société cohésive. Il est de notoriété dans notre pays que le père a de la peine lors du mariage de sa fille, de peur qu'elle ne s'en aille vers une vie qui ne puisse s'apparenter à celle que son père lui a offerte. C'est comment, alors qu'il la voit dans certains cas, elle qui s'est habituée à lui, souffrir de la séparation, qu'il [en vient à] la garder [avec lui].

L'élévation de l'âge de la garde est à 15 ans pour la fille et à 13 ans pour le fils, après quoi ils ont atteint un degré de développement psychique prescrivant de leur laisser le libre choix de vivre chez leur mère ou chez leur père. C'est ce qu'a indiqué l'Envoyé - que Dieu prie sur lui et lui donne la paix quand est venue à lui une femme et qu'elle a dit: ô Envoyé de Dieu:

«Mon époux veut partir avec mon fils alors qu'un puits [qui] arrose ses raisins m'est profitable. L'Envoyé a dit au fils: c'est ton père et c'est ta mère, prends la main de celui des deux que tu veux. Il a alors pris la main de sa mère et elle s'est éloignée avec lui». Merci.

Alors que Wa'd Khaddâm ne faisait allusion au droit islamique qu'incidemment, en complément (salutaire) à la mention du droit moderne, le discours de Hûdâ al-Humsî se situe résolument dans le registre de l'islam et de la sharî‘ $a$. Le dispositif catégoriel est identique, l'apportionnement également, mais la mineure du raisonnement, le registre argumentatif en l'espèce, diffère ou, du moins, fait passer à l'avant-plan un argument qui n'était que marginal chez l'autre orateur. Si bien que le syllogisme prend la forme suivante: (a) l'intérêt de l'enfant est d'être gardé en fonction de ses besoins spécifiques; (b) dans une société islamique, c'est la mère qui assure au mieux la protection des intérêts de l'enfant; (c) le droit

\footnotetext{
4 NdT : Coran, 19:32.
} 
syrien, en tant que droit respectueux des valeurs islamiques de la société, veille à l'intérêt de l'enfant en prolongeant la durée de sa garde par la mère. C'est ici que l'on peut retrouver Elster et sa notion d'argumentation stratégique. Il est en effet clair que, dans les deux discours de Khaddâm et Humsî, l'accent est mis sur la validité du raisonnement avancé. Apparaît toutefois en filigrane l'objectif pratique (et évident dans un cadre parlementaire) d'appuyer le projet d'élévation de l'âge de la garde par la mère et d'ainsi amener une majorité de parlementaires à voter en ce sens. Une assemblée parlementaire est, par définition, composite. Il est bien peu probable qu'elle puisse être acquise unanimement à une seule cause soutenue par une argumentation déterminée unique. Il est en revanche probable qu'il faille trouver le moyen d'amener d'autres composantes du paysage parlementaire à se rallier à la cause que l'on porte. A cet effet, il convient aussi bien de négocier des compromis que d'argumenter stratégiquement. L'un des instruments les plus efficaces semble être, de ce point de vue, l'imposition d'un régime de solidarité négative (Ferrié 2004), c'est-à-dire d'un registre auquel on est astreint de l'extérieur, par le simple jeu d'une énonciation qui le projette à l'avant-plan, mais dont on peut difficilement se soustraire, quelles que soient les préférences personnelles. Dans le débat qui nous occupe, la question de la pertinence du registre islamique n'est jamais posée. Elle est, au contraire, constamment avancée, sur le mode de l'évidence, comme dans l'intervention de Humsî qui voit se succéder des références religieuses donnant au discours la force d'un argument d'autorité (d'autant qu'il n'est personne pour le contester, que ce soit pour des raisons de compétence dans les questions religieuses ou pour des raisons de légitimité confessionnelle), ou sur celui de l'argumentation, comme dans l'intervention de Muhammad al-Habash, que nous reproduisons ci-dessous:

\section{Extrait 19}

149 M. Muhammad Habash -Monsieur le Président - Chers collègues.

L'article 2 de la constitution de la République arabe syrienne stipule que la religion du Président de la République est l'islam. Le deuxième paragraphe de l'article 3 stipule que le droit (figh) islamique est une source principale parmi les sources de la législation.

En réalité, la question de la garde, comme le savent les spécialistes, fait partie des questions sur lesquelles la Loi (sharîa) ultime (khâtima) n'a pas voulu prononcer de propos définitif. Il n'y a pas de texte, ni dans le Saint Coran, ni dans la noble Sunna, qui définisse l'âge de la garde. Dès lors, les vénérables jurisconsultes (fuqahâ') des différentes écoles se sont lancés dans la définition de l'âge de la garde, du choix des Shâfi ites pour [1'âge] de sept ans pour le garçon et dix ans pour la fille au choix des Mâlikites qui ont été jusqu'à dire que la garde du jeune homme [allait] jusqu'à sa majorité et pour la fille jusqu'à ce qu'elle se marie. Al-Hasan al-Basrî a même été au-delà en prolongeant la garde de la fille même si elle se mariait.

De toute manière, les options du droit islamique sont larges sur cette question et je pense que, quand nous ouvrons le corpus de droit islamique dans son ensemble et sans réserve, nous trouvons les options qui conviennent à toute époque et à tout lieu. Pour ce qui est du projet de loi qui est entre nos mains, j'ai participé à la discussion qui a eu lieu à la Commission des affaires constitutionnelles et législatives et j'ai été heureux du respect [manifesté] par tout le monde pour le droit islamique comme source de la législation et de l'attention [portée] aux options des imâms - sur eux soit la satisfaction de Dieu. En fait, le [résultat] auquel la Commission est arrivée 
dans la définition de la durée de la garde à onze ans pour le garçon (ghulâm) et à treize ans pour la fille (fatâ) représente un pas positif. Toutefois, s'agissant du paragraphe b de l'article (le juge a le droit d'étendre la durée de la garde à treize ans pour le garçon et à quinze ans pour la fille s'il lui paraît que l'intérêt de l'enfant gardé l'impose), je propose de ne pas soumettre cette disposition parce qu'elle contraint la femme à en référer au juge pour la prolongation de sa garde des enfants, alors que l'homme est davantage en mesure d'en référer au juge.

Pour cela, je propose que la disposition soit comme suit: (la durée de la garde s'achève quand le garçon a atteint l'âge de treize ans et la fille l'âge de quinze ans). Je pense que cet âge est celui qui correspond aux exigences et besoins qu'a la fille d'être sous la surveillance de sa mère, à cet âge précis, tandis que le garçon a besoin d'être à l'ombre de son père à treize ans pour plonger dans les tribulations de la vie, pour qu'il se prépare et s'entraîne à ses échéances.

Je pense qu'il est utile que la durée de la garde du garçon soit de 13 ans et soit limitée pour la fille à 15 ans, et qu'en référer au juge revienne à l'homme qui est plus apte à cela, sachant que la loi a stipulé les échéances de la garde et qu'il est possible à l'homme de réduire la garde s'il existe des causes impératives. C'est pourquoi j'engage à ce que nous soyons à la recherche de la justice. La justice absolue, c'est quelque chose qui n'existe pas, sauf chez Dieu Tout-Puissant. Nous nous efforçons de la chercher. De nombreuses mères ne peuvent pas veiller sur un enfant plus de deux ans et de nombreux pères ne sont pas en mesure de veiller sur un enfant.

Le Président - interrompant - Cher collègue Muhammad, votre proposition est de supprimer la mention (le juge a le droit d'étendre... etc.) et que [l'article] soit adopté sans elle. Est-ce que ce n'est pas le résumé du propos?

M. Muhammad Habash - poursuivant - Pour que je sois clair, je demande que l'article soit adopté comme suit: (la durée de la garde s'achève quand le garçon a atteint l'âge de treize ans et la fille l'âge de quinze ans) et la suppression complète du paragraphe b. Je marque ma sympathie pour l'opinion de mon ami Monsieur `Abd al-`Azîz al-Shâmî avec qui je suis d'accord quant à cette option. Merci.

L'intervention de Habash se présente comme une véritable argumentation fondée juridiquement. Elle inscrit en effet la légitimité du recours au répertoire islamique dans la Constitution elle-même ${ }^{5}$. En ce sens, elle ne s'impose pas par l'effet d'une solidarité négative, mais par un critère de légalité. Il s'agit donc plutôt d'une solidarité positive, au sens où l'entend Locke, c'est-à-dire d'une solidarité fondée sur la citoyenneté et la moralité (islamique dans notre cas) d'individus œuvrant à la réalisation de l'intérêt général (Ferrié 2006). L'argumentaire de Habash suit le cours suivant: le droit islamique est constitutionnellement une source de la législation syrienne; la question de l'âge de la garde relève du droit islamique, mais celui-ci n'a pas établi de règle intangible en la matière; il est important de légiférer dans le respect de l'islam; toute élévation de l'âge de la garde va dans le bon sens; il faut toutefois tenir compte de l'accès inégal à la

5 Le procédé est subtil. L'article 2 de la Constitution porte sur la religion du chef de l'Etat. Cet article ne figurait pas dans la première version de la Constitution. Il a été ajouté à la suite de violentes émeutes orchestrées par la majorité sunnite. Cette affaire avait fait beaucoup de bruit à l'époque et le nouveau pouvoir avait vite cédé. Il est évident que Habash envoie un signal fort, compris de tous ceux qui se trouvent dans l'enceinte. 
justice de l'homme et de la femme; mieux vaut donc éviter d'avoir à en recourir au juge et élever cet âge à sa limite «naturelle», qui varie entre filles et garçons; l'Assemblée a le devoir de tendre vers la justice, même si cela ne peut être absolu; il faut donc établir des principes, mais aussi faire en sorte qu'ils ne soient pas trop rigides. Habash tend à faire converger légalité et normalité, à donner à son évaluation normative de l'âge normal de la garde des enfants par la mère (15 ans pour la fille, 13 ans pour le garçon) la force d'une loi fondée sur la poursuite de l'intérêt général. C'est en cela précisément qu'il se distingue de l'intervention précédente de Humsî, qui prétendait asseoir son argument dans la seule force de la transcendance. Habash, lui, vise à faire correspondre, dans une démarche jusnaturaliste par excellence, nature, société, loi morale et loi divine.

A la question du Président qui l'interrompt, lui propose une synthèse de ce qu'il vient de dire et lui en demande confirmation (ce qui peut être entendu comme une formule, au sens où l'entend Watson 1979), Habash donne une réponse technique et s'aligne sur la position d'un autre membre du Parlement (Shâmî). Ce dernier ne prend pas part au débat, ce qui laisse supposer qu'il est absent. Que Habash le salue au passage semble également indiquer qu'ils entretiennent des relations de complicité. En faisant mention de l'intervention de cet autre parlementaire, l'intervention de Habash montre comment le débat s'inscrit dans une séquence longue (un «contexte contextualisé»; cf. Dupret 2006) où prennent place d'autres interventions relatives au même objet (qui forment, toutes ensemble, un réseau dialogique; cf. supra). C'est illustratif d'une des multiples façons dont les discours trouvent à se configurer intertextuellement. L'incorporation de la voix d'autrui (Shâmî), d'une tradition textuelle (Hasan al-Basrî), de références dogmatiques abstraites (les Shâfi'ites et les Mâlikites) et de la Constitution permettent à Habash de proposer un discours argumenté dont la validité procède du cumul de ces autorités et de leur agencement.

\section{L'ORDRE EN TANT QUE RESSOURCE: LA PERTINENCE DÉMOCRATIQUE}

Au titre des registres argumentatifs fréquemment utilisés au fil du débat, on relèvera le thème de la démocratie. Ainsi qu'on le faisait remarquer dans une étude précédente, le fait que le débat s'inscrive dans le contexte précis d'une assemblée parlementaire et qu'à ce titre, il soit marqué par la finalité pratique de l'activité législative implique que «la pertinence démocratique l'emporte sur les autres comme ressort d'ordre et impose sa contrainte spécifique à l'interaction » (Ferrié \& Dupret 2004: 275). Cette orientation vers la pertinence démocratique se traduit de différentes manières. D'une part, elle prend la forme de références explicites à l'intérieur de certains tours de parole. D'autre part, elle peut aussi se refléter dans l'usage que les parlementaires font du Règlement intérieur, en tant que norme juridique de régulation des débats et des votes. Parallèlement, les ressources propres à la pertinence démocratique entendue comme ressort d'ordre sont également utilisées comme moyen d'ordre sans préoccupation démocratique. L'ordre intérieur apparaît alors comme un instrument totalement dédié à l'exercice du pouvoir.

Commençons par la production d'ordre dans les débats de l'Assemblée. Nous avons déjà évoqué le rôle pivotal du Président. Il exerce des prérogatives qui sont 
la mise en œuvre de dispositions du Règlement intérieur. Si l'on présente de manière synoptique les deux segments de la paire constitutive du (débat parlementaire), à savoir les [dispositions du Règlement intérieur], d'une part, et l'<application des dispositions du Règlement intérieur>, de l'autre, nous observons comment le phénomène du débat parlementaire relève de ce qu'on appelle, à la suite de Livingston (1995) et Garfinkel (2002), une action instruite. Par le truchement d'un tableau synoptique, nous allons voir comment un ensemble d'instructions, les dispositions du Règlement intérieur en l'espèce, «peuvent être considérées alternativement de telle sorte que la lecture nous révèle un phénomène constitué des deux segments d'une paire: (a) le premier-segment-de-lapaire qui consiste dans l'ensemble d'instructions; et (b) le travail, dans n'importe quel cas réel d'application de ces règles, qui d'une certaine façon transforme le premier segment en une description de la paire» (Garfinkel, 2002: 105-106). Cette paire peut être désignée comme une «action instruite». Le (débat parlementaire), en tant qu'il est une pratique dotée de propriétés phénoménologiques, doit être lu, dans ce cas et ainsi dans chaque cas particulier, comme une paire dont les deux parties, les [dispositions du Règlement] et l'<application des dispositions du Règlement>, sont indissociablement liées:

\section{(DÉBAT PARLEMENTAIRE)}

\section{[dispositions du Règlement]}

Art.39 - A. Personne n'est autorisé à parler sans que le Président ne l'ait autorisé. Si quelqu'un parle sans autorisation, il revient au Président de le lui interdire.

Art. 42 - A. Chaque membre a le droit d'introduire une proposition dans laquelle il demande qu'on confine la discussion ou qu'on l'ajourne.

B. Le Président présente la proposition et il lui revient de donner le droit de parole à l'auteur de la proposition, à une personne la soutenant ou à l'un de ses opposants. Ensuite, il la met au vote.

C. L'acceptation de la proposition est conditionnée par l'accord de la majorité des membres présents.

<application des dispositions du Règlement>

Le Président

47 Vous avez entendu le rapport. Le rapport et le projet de loi sont 48 présentés à la discussion générale. Ceux qui désirent parler le 49 montrent en levant la main. Pendant que Monsieur le greffier enre50 gistre les noms de ceux qui désirent parler, j’ai le plaisir de saluer

51 [notre] collègue Monsieur Nizâr al-'Assasî, ministre de la Justice,

52 qui est sorti de nos rangs et occupe le siège du troisième pouvoir.

53 Nous luis souhaitons la réussite dans son travail. Nous espérons

54 que le pouvoir judiciaire devienne d'une blancheur immaculée. A

5 présent, la parole est à [notre] collègue Wa'd Khaddâm.

M. Ahmad Ghuzayl

238 - Monsieur le Président - Chers membres [du Parlement] (al-sâda

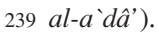

240 J'ai présenté une proposition écrite au bureau de la présidence, sur 241 la base de l'article 42 du Règlement intérieur, pour que l'on 242 verrouille le débat et que l'on procède à la discussion des articles 243 du projet de loi. Je souligne que toutes les propositions que les 244 collègues ont présentées sont des propositions de valeur, mais 245 elles tournent toutes autour de l'amendement de tel ou tel article.

246 Je considère qu'il serait plus approprié de procéder à la discussion 247 du projet de loi article par article. Merci.

Le Président

248 - Ceux qui sont d'accord avec la proposition de [notre] collègue 249 Ahmad Ghuzayl le montrent en levant la main / les mains ont été 250 levées / majorité, accepté, nous verrouillons le débat.

251 A présent, ceux qui sont d'accord pour que l'on procède à la 252 discussion du projet de loi article par article le montrent en levant 253 la main / les mains ont été levées / majorité, Monsieur le greffier 254 lise à haute voix l'article premier. 


\author{
Art.43 - $\quad$ A. On ne \\ peut adresser la parole \\ qu'au Président ou à l'As- \\ semblée. \\ C. L'orateur n'est jamais \\ interrompu. On ne peut \\ parler d'un seul sujet plus \\ de trois fois, sous réserve \\ des dispositions du \\ paragraphe B de l'article 40 \\ de ce Règlement.
}

\footnotetext{
M. `Abd al-Qâdir Hins

458 - Poursuivant - Toute ma réflexion tourne autour de ce sujet,

459 parce que la non-contradiction de la loi que nous promulguerons

460 avec les autres lois, c'est le devoir de l'Assemblée. Et la loi d'au-

461 jourd'hui contredit l'un des textes du Code de statut personnel.

Le Président

462 - Interrompant - [Cher] collègue, à mon avis, mais je ne suis pas

463 avocat, je comprends la [question] posée comme étant numérique.

464 L'article dit: La garde s'achève de telle façon et dans des condi-

465 tions présumées précises. Nous n'allons pas entrer dans la ques-

466 tion de savoir s'il y a des interdits ou etc. De toute manière, si

467 vous voulez inclure ce texte dans votre proposition, incluez-le.

M. `Abd al-Qâdir Hins

468 - Poursuivant - Je me contenterai de lire à haute voix ma propo469 sition, parce que [...]
}

On peut, à la lecture de ce tableau, prendre la mesure du caractère instruit de l'action du Président. On voit en effet à quel point elle s'inscrit dans un cours contraint et normé par le Règlement intérieur, bien que cela ne signifie nullement qu'il soit complètement déterminé par ses dispositions, comme en attestent les lignes 461-465 où le Président interrompt l'orateur en dépit de la stipulation expresse de l'article 43-C. En d'autres termes, le Règlement intérieur agit comme un guide pour l'action, même quand il n'est pas invoqué explicitement. L'action du Président et, au-delà, le débat parlementaire prennent place dans un contexte balisé par un ensemble de référents dont la pertinence est rendue explicite par les orientations des intervenants. Si l'action est instruite, le contexte est donc, pour sa part, contextualisé (Dupret 2006). Il s'inscrit dans une séquence longue; il prend place dans un dispositif procédural et référentiel qui lui préexiste et qui agit sur sa configuration. Mais cette action est également instructrice et le contexte, contextualisant. Cela signifie que chaque nouveau développement du débat actualise celui-ci, en fait une nouvelle instance explicite du genre «débat parlementaire», contextualise toute action qui surviendrait dans son cours. Par ailleurs, chaque développement nouveau, en tant que deuxième partie de la paire ([règle]$<$ pratique de la règle $>$ ), instruit à son tour les pratiques ultérieures qui s'appuieront sur la récurrence des manières de faire pour produire et reproduire l'usage procéduralement correct du Règlement intérieur. Normé par le Règlement et les pratiques passées, le débat en devient à son tour normatif quant à ses pratiques futures.

La présidence, en tant qu'action instruite, est déterminante dans la production et la gestion de l'ordre au sein de l'Assemblée. En s'appuyant sur le Règlement et en en orientant la lecture, le Président exerce son contrôle sur le déroulement des débats. La procédure, telle qu'orchestrée par lui, apparaît ainsi comme un instrument d'exercice du pouvoir. Dans sa gestion d'opérations qui peuvent sembler n'appartenir qu'à la «cuisine interne » de l'Assemblée, le Président procède à une orientation et à une canalisation très déterminées. Il attribue systématiquement les tours de parole, il interrompt, il contredit, il abrège, il synthétise, il formule, il ironise, il fait procéder à la lecture des rapports et des projets, il commande les opérations de vote, toutes choses que nous avons déjà eu l'occasion de passer en revue. Nous nous contenterons donc de reproduire un extrait dans lequel, jouant d'ironie, le Président réitère sa conception limitative de l'objet du débat et, ce 
faisant, non seulement cherche à en abréger la durée, mais dans le même mouvement à en limiter la portée.

\section{Extrait 20}

Le Président
- Interrompant - [Chère] collègue, s'il vous plaît, nous sommes sur un sujet très précis - comme nous l'avons dit précédemment - nous discutons de l'article 19 de la loi 24 , nous ne sommes pas [en train de discuter] des lois égyptiennes ni du droit islamique ni d'aucun autre sujet. C'est pourquoi je demande que vous restiez dans les limites du sujet. Nous discutons de la question telle que proposée. La Commission et le gouvernement qui ont étudié le projet ne sont pas des enfants. Il ne fait aucun doute qu'ils ont entrepris d'étudier les Etats environnants et le droit [islamique], qu'ils ont mené toutes les études, ont mené des consultations et ont posé des questions. Il n'est pas possible à ce stade que nous leur demandions au dernier instant d'en revenir à des données de base. Je répète donc, [chère] collègue que nous [devons] discuter de ce sujet dans les limites de l'article et de son amendement.
- Poursuivant - Je suis pour la proposition du docteur Muhammad Habash et je ne suis pas pour le rapport de la Commission. Merci.

Cette interruption et l'ironie qui la traverse ont pour propriété de menacer la crédibilité de l'orateur. La notion d'action-menaçant-de-faire-perdre-la-face (Brown \& Levinson 1987) s'appuie sur la conception goffmanienne de l'interaction en face-à-face (Goffman 1981) et sur les expressions de sens commun qui y sont associées («garder» ou «perdre la face»). Elle vise à saisir les implications émotionnelles et interactionnelles de la prise de parole en public et des réactions qu'elle enclenche. En interrompant le tour de parole de l'orateur, le Président de l'Assemblée manifeste clairement qu'il le considère comme superflu, ce qui en attaque la crédibilité. En ironisant sur le fait qu'on discute de droit syrien et non de droit égyptien ou islamique, le Président justifie son interruption et en redouble l'effet décrédibilisant, accusant implicitement l'orateur de faire perdre son temps à l'Assemblée. L'effet de l'interruption est immédiat: l'orateur formule en une phrase courte son soutien à la proposition Habash et son opposition à la proposition de la Commission. Autrement dit, l'interruption et la menace qu'elle a fait peser sur l'orateur amène cette dernière à immédiatement tenter de réparer l'atteinte portée à sa crédibilité en se conformant en tous points aux exigences de concision et de précision formulées par le Président. Cet épisode, parmi bien d'autres, permet de discerner avec précision comment l'ordre est produit et le pouvoir exercé, au sein de l'Assemblée, à l'aide des prérogatives procédurales inscrites dans le Règlement intérieur et mises en œuvre par le Président, dans une action faite indissolublement de la règle et de son application.

La question de l'ordre peut également apparaître comme une thématique relevant de la pertinence démocratique. Elle prend alors la forme, à l'intérieur de certains tours de parole, de références explicites à des sujets tels que les droits de la majorité, le respect des minorités, la relation entre les trois pouvoirs ou l'égalité des sexes. Dans ce cas, la pertinence démocratique est utilisée comme ressort d'ordre, c'est-à-dire comme principe régulateur de la prise de décision au sein de l'Assemblée. 


\section{Extrait 21}

Dans cet extrait, l'orateur invoque la démocratie en tant que système délibératif. Le principe de la discussion des projets de loi doit l'emporter sur le respect des procédures formelles (le verrouillage du débat). En même temps, le respect d'autres procédures (la lecture à haute voix des projets) garantit la bonne mise en œuvre de cette démocratie délibérative. La réponse du Président consiste à rappeler l'organisation séquentielle des opérations. Il reconnaît en quelque sorte le registre argumentatif utilisé par l'orateur dans son tour de parole, mais il en corrige les défauts techniques et procéduraux. S'il dénie à cette intervention le statut de point d'ordre, ce n'est donc pas en tant qu'il serait opposé à sa pertinence de fond, mais seulement à sa pertinence de forme. Plus loin dans le débat, d'ailleurs, c'est le même Président qui recourt à la pertinence démocratique:

\section{Extrait 22}

Monsieur le Président - Chers collègues.

Le vote de cette manière n'est pas autorisé. Je siège à cette assemblée depuis 15 ans et le vote ne s'obtient pas de la sorte. Il faut que le membre se lève et qu'il dise je suis pour ou contre. Merci.

[Cher] collègue, nous avons entrepris de compter ceux qui sont d'accord. Ils sont au nombre de 76 membres. Pour la démocratie, nous allons entreprendre de compter ceux qui ne sont pas d'accord. Ceux qui ne sont pas d'accord le montrent en levant la main (quelques mains sont levées). Il y a ici un point d'ordre de [notre] collègue Muhammad al-Satam. Il a la parole.

Monsieur le Président - Chers collègues.

Cette année est la neuvième année pour moi dans cette assemblée et je n'ai jamais vu un vote de cette manière. Si l'on vote deux fois ou trois, ce n'est pas logique et cela conduit à des embrouilles.

[Cher] collègue, c'est parce que l'affaire s'est embrouillée que nous avons demandé le nouveau vote. Vous vous êtes porté votre propre critique. La parole est à [notre] collègue 'Abd Allâh al- Mawsallî.

Dans la bouche du Président, la pertinence démocratique ne recouvre toutefois plus la politique délibérative, mais le principe majoritaire et, partant, l'exactitude du comptage des voix. La situation étant devenue confuse - tout le monde en convient -, il veut s'assurer du décompte. C'est l'effet contraignant de la pertinence démocratique, en tant que ressort d'ordre, qui émerge avec vigueur: 'Abd 
al-Rahmân al-Ahmar fait usage de cette pertinence pour contester la procédure de vote qui a été suivie; le Président accède à la critique et recourt explicitement à cette pertinence démocratique pour faire procéder à un nouveau vote; Muhammad al-Satam l'invoque à son tour implicitement pour s'étonner de la façon de procéder; et le Président, tout en faisant porter la responsabilité du cafouillage sur le caractère procédurier de certains orateurs, réitère son alignement sur cette pertinence. Notons au passage que le Président utilise à nouveau l'ironie pour retourner la critique et maintenir l'ordre du débat. Il accuse en effet Muhammad al-Satam de s'interroger sur un problème à l'origine duquel il se trouve, lui, et par derrière, les autres membres de l'Assemblée qui ont soulevé des points de procédure. La force du registre de l'ironie (et de la menace qu'elle fait peser sur la «face» de celui qu'elle vise) est à nouveau manifeste.

C'est aussi le thème de la séparation des pouvoirs qui revient dans les usages de la pertinence démocratique:

\section{Extrait 23}

$\begin{array}{ll}\text { M. `A.A. al-Mawsallî } & \text { - Monsieur le Président - Chers collègues. } \\ & \text { Il est vrai que l'article 42 du Règlement intérieur permet à chaque membre } \\ \text { de demander le verrouillage du débat et il revient à l'Assemblée à sa majo- } \\ \text { rité d'en décider ainsi, mais le verrouillage du débat est une chose et l'audi- } \\ \text { tion du pouvoir exécutif une autre chose. [Nous faisons] comme si le } \\ \text { ministre de la Justice n'était pas présent et nous le considérons comme un } \\ \text { membre et n'entendons pas son avis avant le procéder à la discussion des } \\ \text { articles. [Notre] collègue Muhammad a proposé l'amendement de l'article, } \\ \text { quel est l'avis du pouvoir exécutif à ce propos? Merci. }\end{array}$

La démocratie délibérative est à nouveau mise en avant, dans l'intervention de Mawsallî, en ce sens qu'il se fait l'avocat d'un débat informé. C'est aussi le principe des trois pouvoirs et de leur collaboration qui le motive, quand il demande à ce que l'initiateur du projet, à savoir le pouvoir exécutif en la personne du ministre, fasse connaître son opinion sur les changements que l'on suggère d'apporter à son texte. Fidèle à sa conception plutôt expéditive des débats, le Président semble trouver cette demande d'opinion superflue, mais il ne s'y oppose pas, au motif précisément qu'une question controversée mérite d'être instruite. Le ministre de la Justice, quand vient son tour, s'en tient à une stricte séparation des pouvoirs, laissant entendre que le travail du ministère s'arrête au seuil de l'Assemblée. Le dernier orateur semble intervenir comme un carabinier d'Offenbach, à contre- 
temps. En somme, cet extrait indique la propension des orateurs à s'appuyer sur des principes relevant de la pertinence démocratique qui, indépendamment des préférences individuelles, fait de ce groupe une assemblée parlementaire.

La pertinence démocratique en tant que ressort d'ordre a ceci de particulier qu'elle exerce une sorte de prééminence sur les autres registres argumentatifs ou pertinences mobilisés au cours du débat. Parce que la pertinence démocratique est au principe même de l'instance à l'intérieur de laquelle ce débat prend place, elle s'impose comme une contrainte aussi bien substantielle que procédurale. Contrainte substantielle, d'une part, en ce sens qu'elle fait écho à des principes démocratiques dont personne ne peut se dédire, même si la conviction demeure éventuellement qu'il s'agit là d'un jeu de façade. Contrainte procédurale, d'autre part, en ce sens que la marche à suivre ne peut s'afficher que comme légaliste, quand bien même l'on chercherait mille et un moyens de la contourner et de la vider de son contenu.

D'une certaine façon, l'orientation des parlementaires vers la pertinence démocratique se reflète également dans l'usage qu'ils font du Règlement intérieur, en tant que norme juridique de régulation des débats et des votes. Loin de n'être qu'une norme déterminant strictement l'action, le Règlement est utilisé comme ressource, comme moyen d'intervention dans le débat. En tant qu'argument d'ordre, légitime et légal, il fonctionne comme un espace d'opportunité dans lequel les participants peuvent s'introduire en sorte d'accomplir la tâche que leur mandat leur assigne et qu'ils entendent accomplir, c'est-à-dire «agir-comme-unparlementaire».

Parmi les différentes ressources offertes par le Règlement intérieur, la règle du verrouillage occupe, dans le débat qui nous concerne, une place de choix. Nous avons déjà eu l'occasion d'en traiter plus haut. Il suffira donc à présent de souligner que cette technique offre la possibilité de couper court à la discussion générale et d'en passer directement à la discussion par article et au vote des différents projets et propositions. Dès lors que son adoption est soumise au vote de l'Assemblée, le verrouillage offre la possibilité de confiner le débat dans une direction favorable à la majorité des membres de l'Assemblée. En temps normal, dans le contexte parlementaire syrien, il s'agit donc d'une arme très efficace aux mains du régime, dans la mesure où celui-ci dispose d'une majorité écrasante. En revanche, dans des situations où ce ne sont pas les appartenances politiques qui sont déterminantes et où apparaissent d'autres clivages, tels le sexe et la confession, le verrouillage se présente davantage comme la possibilité procédurale d'imposer la prise en considération de propositions alternatives à celles qui, bien qu'émanant du gouvernement, ne procèdent pas de la prééminence de l'Exécutif.

\section{Extrait 24}

590 Le Président
- [Chers] collègues, il y a une deuxième proposition de verrouillage du débat. Ceux qui sont d'accord avec cette proposition [le] montrent en levant la main - les mains sont levées - majorité favorable à la proposition. [Chers] collègues, le règlement intérieur stipule que nous soumettions [au vote] l'article tel qu'amendé par la Commission. S'il n'emporte pas la majorité, nous soumettons [au vote] les autres propositions. Ceux qui sont d'accord avec l'article tel que présenté dans le rapport de la Commission [le] montrent en levant la main - les mains sont levées - minorité. La parole est à [notre] collègue Muhammad Habash. 


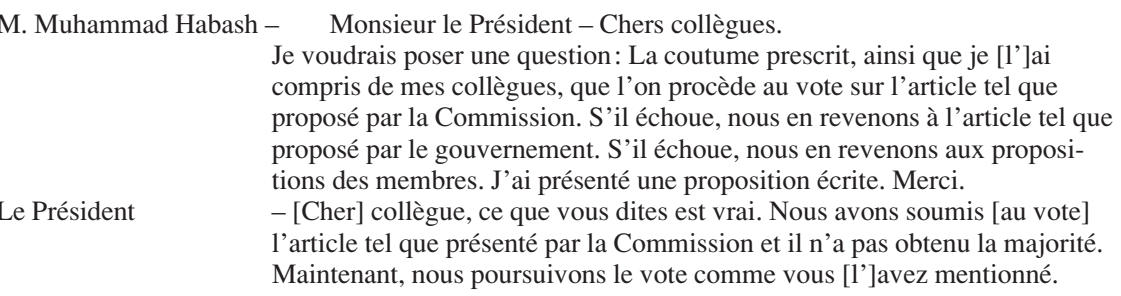

Ce n'est pas que l'article 42 du Règlement intérieur et la règle du verrouillage qu'il stipule que les parlementaires utilisent comme ressource d'intervention procédurale dans le débat. Les règles de quorum et de vote font l'objet de très nombreuses discussions, sous la forme de points d'ordre:

\section{Extrait 25}

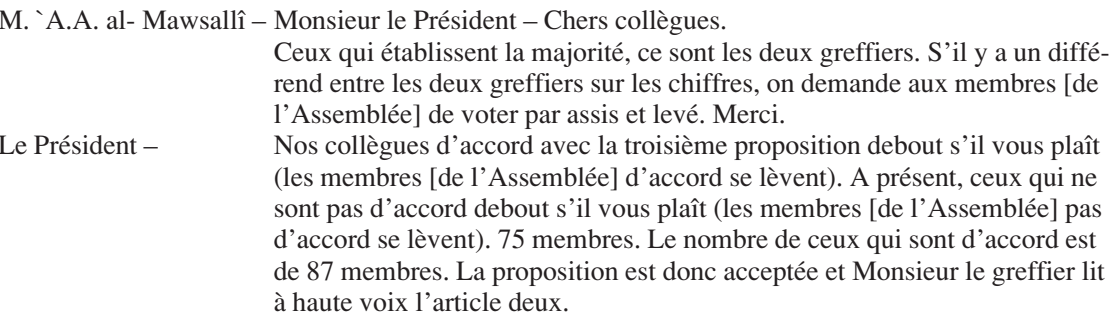
rend entre les deux greffiers sur les chiffres, on demande aux membres [de l'Assemblée] de voter par assis et levé. Merci.

Le Président - $\quad$ Nos collègues d'accord avec la troisième proposition debout s'il vous plaît (les membres [de l'Assemblée] d'accord se lèvent). A présent, ceux qui ne sont pas d'accord debout s'il vous plaît (les membres [de l'Assemblée] pas d'accord se lèvent). 75 membres. Le nombre de ceux qui sont d'accord est de 87 membres. La proposition est donc acceptée et Monsieur le greffier lit à haute voix l'article deux.

Cette préoccupation pour les règles formelles relève de ce que nous avons appelé par ailleurs l'orientation vers la correction procédurale (Dupret 2006). Une bonne partie de ces interventions procèdent en effet d'un souci d'expression de l'accomplissement de la fonction de parlementaire dans les formes requises, dans les règles de l'art. En soulevant des points d'ordre, les orateurs manifestent le fait qu'ils agissent en tant que parlementaires et qu'ils comprennent cette fonction de manière légaliste. Il s'agit pour eux d'attester, face à une audience matérielle et virtuelle, un respect des procédures qui, seules, garantissent la validité des législations adoptées. Il s'agit également pour eux d'attester de leur compétence et de leur professionnalisme.

Le souci de la forme est cependant tellement marqué, dans ce débat parlementaire, qu'on est en droit de se demander s'il ne déborde pas l'orientation vers la correction procédurale et ne traduit pas aussi un activisme protestataire de type civique, à l'image de la grève du zèle, c'est-à-dire d'une surenchère procédurière visant à faire valoir un point de vue oppositionnel de l'intérieur même d'un système majoritaire ou unanimiste. Il s'agit en quelque sorte de menacer la mécanique de paralysie à partir de ses propres règles de fonctionnement, non pas pour mettre en cause les règles elles-mêmes, mais parce qu'elles constituent un dernier recours possible quand la substance du débat et son issue sont en général largement préjugées. Le souci de correction procédurale, dans pareil cas, ne répond plus à une orientation bureaucratique vers l'accomplissement normal et normé des choses, mais devient un instrument d'action dans le processus parlementaire ordinaire et sur celui-ci. 


\section{Extrait 26}

707 Le Président 708 M. Ghâlib `Unayz -

Monsieur le Président, chers collègues.

J'ai une question sur le nombre des [membres de l'Assemblée] inscrits présents au début de la séance. Si le nombre de ceux qui sont d'accord est inférieur à la moitié, le projet n'est pas considéré comme décidé. C'est pourquoi je demande [à connaître] le nombre des [membres de l'Assemblée] inscrits présents au début de la séance. Merci. La parole est à [notre] collègue Ghâlib `Unayz.

Monsieur le Président, chers collègues.

Selon le règlement intérieur, lorsque l'accord est obtenu sur un projet de loi ou sur un article, il convient que soit évalué le nombre des [membres] présents et que les votants en faveur de l'accord s'élèvent à la moitié plus un pour l'obtention de la majorité. Tout vote en contravention avec cela est considéré en contravention avec le règlement et nous exposera à une difficulté future. Je sollicite que l'on s'assure de ce texte et que l'on soit éclairé de manière nette et complète pour que nous ne exposions pas à une difficulté juridique par rapport au règlement intérieur et à la constitutionnalité de cette loi lorsqu'elle sera promulguée à l'avenir. Merci.

La parole est à [notre] collègue `Âdil Jâmûs.

Monsieur le Président, chers collègues.

La séance est considérée comme réglementaire lorsque la moitié du nombre absolu des membres plus un, c'est-à-dire 126 membres. 87 membres. 87 membres est calculé sur 126 et pas sur 250. Merci.

[Cher] collègue Ahmad, j'ai dit est-ce qu'il y a des commentaires sur le projet de loi, pas que le ministre soit interrogé. Pour cela, j'espère que vous reporterez votre commentaire. [Cher] collègue Khidr, le nombre des membres d'accord est de 87 et le nombre des membres qui ne sont pas d'accord est de 75. Personne ne s'oppose sinon vous. Et, [cher] collègue `Âdil et [cher] collègue Ghâlib, chacun de vous deux présente un avis différent sans objection. La parole est [notre] collègue Khidr al-Nâ im.

M. Khidr al-Nâ`im - Monsieur le Président, [chers] collègues.

Il y a ici un point de règlement. Je suis en désaccord avec [notre] collègue ‘Âdil, [notre] grand frère. L'article 67 stipule: «L'Assemblée prend ses décisions et adopte les lois sur le principe de la majorité des [membres] inscrits présents au début de la séance. Sont exceptées de ceci les questions pour lesquelles la Constitution ou le règlement intérieur a défini une majorité précise».

Dès lors, il faut que le nombre de ceux qui sont d'accord soit de la moitié du nombre des inscrits au début de la séance plus un, de telle sorte que le vote soit considéré comme légal et valide. Merci.

[Cher] collègue, celui qui a quitté la salle est-il considéré comme s'abstenant du vote ou n'en est-il pas ainsi, [cher] collègue? La parole est à [notre] collègue `Abd Allâh al-Mawsallî.

M. `A.A. al-Mawsallî - Monsieur le Président - Chers collègues.

Ce que [notre] collègue Khidr a eu l'amabilité [de dire] est exact. L'article qu'il a cité est également exact. Mais je m'étonne de ce commentaire et de cette question. J'ai présidé de nombreuses séances quand j'étais vice-président et nous avons adopté des lois en présence de quarante membres. A présent, pourquoi cette agitation à ce sujet? Merci.

Le Président - $\quad$ Ceux qui sont d'accord avec l'ensemble du projet de loi le montrent en levant la main (les mains sont levées), majorité. Le projet est adopté et il devient loi.

Le Règlement intérieur et ses dispositions procédurales apparaissent, dès lors, comme un moyen argumentatif puissant dont disposent les parlementaires, un 
moyen qui leur ouvre un espace de manœuvre et de contestation. Il s'agit d' «une utilisation détournée de ressources mises en place par le système politique luimême» (Elster 2005: 60). Plus exactement, il s'agit de l'utilisation à outrance du dispositif procédural dans le but de perturber le système ou, tout au moins, de lui faire «payer le prix » du rôle marginal dans lequel il tient ses propres acteurs. Dans la typologie de Hirschman, cela correspondrait à une stratégie de prise de parole, c'est-à-dire de critique de l'intérieur même du système. En l'espèce, cette prise de parole ne prend pas tant la forme d'une contestation ouverte que celle d'une stratégie d'exaspération, par le recours outrancier aux règles de procédure que le système offre lui-même et auxquelles, partant, il ne peut se soustraire.

\section{CONCLUSION}

Une des thèses importantes de cet article est que le vote d'une loi portant amendement du statut personnel est étroitement tributaire du contexte institutionnel de son adoption et que les règles procédurales constituent une ressource majeure entre les mains des participants à ce processus. Autrement dit, les institutions et les procédures ne représentent pas seulement des règles et des contraintes qui s'imposent aux membres de l'Assemblée, mais aussi des moyens dont disposent les participants et vers lesquels ils s'orientent dans le cours de leur activité spécialisée.

Etudier l'adoption d'une loi en tant que pratique suppose d'observer le fonctionnement d'institutions représentatives. Celui-ci procède à deux niveaux : d'une part, l'appareil de désignation des représentants et, de l'autre, le processus de délibération au sein de l'Assemblée. Si l'on s'en tient au second de ces niveaux, on remarque que les délibérations sont marquées du sceau de l'incertitude, dès lors qu'elles impliquent la coopération de gens qui agissent d'une manière personnelle, elle-même produit de causes multiples, au titre desquelles des motifs personnels, des calculs politiques, des contraintes contextuelles, des relations personnelles, etc. Cela vaut aussi dans un régime autoritaire, où le gouvernant ne sait jamais jusqu'à quel point il peut se reposer sur des gens occupant des positions constitutionnellement et juridiquement importantes et lui obéissant, non en raison de la loi, mais par intérêt ou par servilité. La création d'institutions représentatives a pour effet de multiplier les moyens infra-décisionnels par lesquels les membres du Parlement peuvent prendre des initiatives correspondant à ce qu'ils pensent être acceptable pour le gouvernant et conforme à leurs compétences. Ces gens envisagent leurs compétences à partir de dispositifs catégoriels grâce auxquels ils dessinent la frontière de ce qui est permis à l'intérieur de ce cadre institutionnel. Cela laisse une grande marge d'incertitude. En outre, dès lors que, formellement, le pouvoir est dérivé de la volonté du peuple, l'expression de cette volonté doit de ce fait être, formellement aussi, tangible et incarnée dans l'activité de l'assemblée législative. En termes praxéologiques, cela signifie que même les gouvernants autoritaires s'orientent vers la légitimité populaire en tant que source de leur droit à gouverner, ce qui implique le recours à l'expression continuelle de l'assentiment du peuple au travers d'une institution qui en est le garant et le rend explicite, à savoir le Parlement. A ce titre, que le régime soit démocratique ou autoritaire, les activités parlementaires forment l'arrière-plan normal sur lequel 
opèrent les différentes attentes, anticipations, catégories et modes de cognition politique.

Dans ce contexte pratique, les intervenants recourent à différents registres argumentatifs, ce que nous avons appelé des pertinences. Il en est une qui occupe une place particulière, du simple fait qu'elle est au fondement même de l'institution parlementaire et de son fonctionnement. Il s'agit de la pertinence démocratique qui, outre sa capacité à être utilisée pour nourrir des arguments de fond, sert aussi de ressort d'ordre, c'est-à-dire de principe régulateur des activités qui prennent place dans l'enceinte. Alors que le recours aux autres pertinences ressortit souvent de la solidarité négative, c'est-à-dire qu'il positionne le débat sur un registre auquel les participants ne peuvent se soustraire, la pertinence démocratique comme ressort d'ordre appartient davantage au régime de la solidarité institutionnelle, en vertu de laquelle il est impossible de fonctionner normalement dans une institution telle que le Parlement sans admettre les règles de base de son fonctionnement. Se pose alors la question de l'expression de l'accord, du désaccord, du consensus et de l'opposition. Dans le cadre précis du débat que nous avons étudié, le projet de loi présenté par le gouvernement et l'amendement proposé par la Commission des affaires constitutionnelles et législatives étaient loin de faire l'unanimité. Pour exprimer leurs positions respectives, les parlementaires sont intervenus en respectant la lettre du Règlement intérieur, jusqu'à l'excès de zèle. Il s'agissait tantôt de s'aligner sur les positions du gouvernement ou de la Commission, tantôt (et même souvent) de s'y opposer. Dans ce dernier cas, sous l'effet du contexte parlementaire et de la solidarité institutionnelle, l'opposition s'est faite sur le mode obligé de la pertinence démocratique. Autrement dit, l'expression du désaccord s'est faite au travers de la réaffirmation d'un consensus autour de l'institution et de son mode de fonctionnement. Partant, si désaccord il y eut, ce fut à l'intérieur d'un cadre procédural contraignant qui en neutralisait le caractère subversif: ce n'était pas le lieu d'une opposition au régime, mais seulement un moment où a trouvé à s'exprimer l'opposition du régime.

Nous n'entendons bien sûr pas nier la nature spécifique des assemblées parlementaires de pays comme la Syrie. Nous ne prétendons pas non plus que ces assemblées soient élues au terme d'un processus transparent et démocratique. Aucun observateur de la vie politique de ce pays ne peut ignorer le dévoiement du principe d'élections libres et équitables. Il n'en reste pas moins que l'Assemblée du Peuple travaille et produit un corpus de textes législatifs d'une façon largement déterminée par l'environnement institutionnel dans lequel le processus se déploie. En d'autres termes, qu'il soit vertueux ou vicieux, il existe un cercle dans lequel les activités législatives se trouvent aspirées du simple fait qu'elles doivent prendre place au sein d'un certain type d'institutions générant ses règles de procédure, son allocation des tours de parole, ses processus de vote, son formalisme, son organisation propre des débats, ses asymétries locales et politiques, etc. C'est à ce niveau que, loin de n'être que des contraintes s'imposant aux participants au débat, les caractéristiques institutionnelles des assemblées législatives offrent également des ressources dont leurs membres font fréquemment usage pour faire valoir un point, vocaliser (au sens de voicing) une préférence, affirmer leur désaccord ou faire connaître leur point de vue à une audience élargie. Comme l'affirme Jon Elster (1998), une institution démocratique comme le Parlement exerce un 
effet sur les régimes politiques, même autoritaires, parce qu'elle induit un certain nombre de comportements. C'est en cela que la démocratie s'inscrit dans la pratique de la démocratie, et non dans la préexistence de mœurs ou de convictions démocratiques.

En nous intéressant à la production pratique et contextuelle de la norme dans l'enceinte parlementaire syrienne, nous avons pu saisir comment s'établit une relation entre le cours situé d'un débat et le jeu des opinions virtuelles postulées par les intervenants dans cette enceinte. Il s'agissait d'établir une relation documentable d'un point de vue praxéologique entre la finalité pratique du travail parlementaire (voter une loi, dans notre cas), des choix normatifs issus d'un certain type de contraintes organisationnelles propres à un site dialogique et l'inscription de ces choix dans le contexte plus large d'un réseau dialogique. Nous avons également pu voir comment l'action est finalisée, contrainte et destinée à être intelligible. Finalité, contrainte et intelligibilité procèdent de ce que les pratiques humaines s'appuient sur les pratiques humaines.

Quant aux choix normatifs exprimés par les intervenants, ils doivent être considérés comme des préférences qui se stabilisent dans le cours des interactions et non comme des réalités constituées ex ante. Ainsi, ce qui stabilise les préférences ne réside pas dans leur transcendance, mais dans leur publicité — c'est-àdire dans leur circulation et leurs reprises par autrui, dans des versions similaires (isomorphes) ou contrastives (allomorphes). L'affirmation d'une préférence, dans le cours d'un débat, dépend, dans un premier temps, de ce que les gens peuvent penser qu'autrui partage leurs postulats et agit selon les procédures pratiques de la raison mondaine. Elle dépend, dans un second temps, de leur capacité à s'ajuster à ce qu'ils sont conduits à penser être les positions d'autrui, du fait de la manifestation publique de ces positions lors de l'interaction. Ces ajustements ne peuvent bien évidemment pas être tenus pour mécaniques: la communication n'est exempte ni de bégaiements ni de balbutiements, et les réparations qui surviennent à la suite de ces échecs sont là pour attester de ces aléas et de ce que l'ordre social, auquel la communication contribue activement, n'est pas sans incertitudes.

Engagés dans la fixation de normes et dans la production de lois, les membres d'une assemblée parlementaire procèdent collectivement à la formulation implicite ou explicite de dispositions d'ordre éthique dans un environnement de procédure et de pertinence particulier, dans un cadre de normalité de raisonnement et d'agissements situés, au milieu d'un dispositif complexe de catégorisations et de thématisations possibles. Les multiples agencements de ces environnements, cadres et dispositifs ne sont intelligibles qu'à la condition de les replacer dans le cours contextuel et séquentiel de leur accomplissement empirique. Toutefois, ces contraintes intrinsèquement liées à l'organisation interne du travail parlementaire se doublent de contraintes propres à la relation que les législateurs entretiennent publiquement avec autrui du fait même de cette organisation. En effet, lors d'un débat parlementaire, ceux-ci s'orientent vers un public présent, dans l'enceinte parlementaire, mais aussi vers un public absent: les téléspectateurs, leurs électeurs, les journalistes, les personnes qu'ils évoquent dans leurs propos, les autorités politiques du pays dont ils dépendent plus ou moins selon la nature du régime. Ces publics n'interagissent pas, en ce sens qu'ils ne sont pas en coprésence. Cependant, leurs propos, leurs prises de positions, leurs arguments sont séquentiellement organisés avec les propos tenus par les parlementaires, dans la 
mesure où ils se nourrissent les uns des autres. En ce sens, la délibération parlementaire structure une organisation sociale contingente et provisoire. S'il est difficile de savoir si les publics vers lesquels s'orientent un parlementaire existent ailleurs que dans son esprit, le repérage des reprises de ses propos indique, en revanche, ceux qui s'auto-constituent comme publics concernés, de même que les formes plus ou moins contrastives de ces reprises nous renseignent sur les caractéristiques de ces publics. Elles nous renseignent aussi sur la pertinence des propos, sur leur recevabilité. Inversement, les reprises, par les parlementaires, de propos tenus à l'extérieur de l'enceinte du Parlement indiquent quels publics sont pris aux sérieux, ménagés, et jusqu'à quel point, ou au contraire, dénigrés. C'est ici que prend place la question du «dénombrement» des opinions.

Les opinions sont constituées des reprises contrastives des termes d'une délibération articulée à des collectifs. Si ces opinions comptent, c'est bien parce que les différents protagonistes d'un débat - qu'ils soient situés dans un site dialogique ou dans son réseau - sont censés faire nombre. Faire nombre, dans une organisation dont le cœur est un Parlement, cela veut dire entrer dans la constitution de majorités. Est-ce à dire que la norme est laissée à l'opinion du plus grand nombre, comme le soutiennent, depuis Platon, les critiques de la démocratie? Non — et c'est ici que le retour praxéologique sur l'organisation de la délibération dans les enceintes parlementaires s'avère tout à fait irremplaçable, puisqu'il montre comment la sélection d'une version, d'une norme, est connectée, certes, au souci des opinions dénombrées, mais que cette connexion est soumise aux contraintes intrinsèques de l'ordre parlementaire et qu'il en ressort tout autre chose que la simple soumission à ces opinions ou à la règle majoritaire qui ferait procéder l'adoption d'une norme d'un simple fait mathématique. Profondément, la délibération parlementaire est une activité sociale qui met, au contraire, les choix normatifs à l'abri d'une influence directe de l'opinion et qui interdit, en même temps, aux législateurs de méconnaître les inclinations de leurs constituants.

Bien que l'on se soit attaché à analyser un débat ayant pris place dans un pays où l'organisation parlementaire s'inscrit dans des pratiques autoritaires et où il existe des limites fortes à l'influence des constituants du législateur sur la fixation des normes et la fabrication des lois, il serait faux de croire qu'il n'est pas tenu compte de leur existence et de leur participation au jeu politique. Autrement dit, les délibérations parlementaires sont, dans ce contexte, un cas de figure important, si pas principal, de la constitution d'équilibres politiques complexes dépassant le seul «fait du prince» et, partant, des relations d'autonomie et de dépendance dans lesquelles l'Assemblée, y compris le Parti au pouvoir qui y est surreprésenté, et le prince se trouvent engagés.

Baudouin Dupret,dupret@link.net Belhadj Souhaïl, babjdid@yahoo.fr Jean-Noël Ferrié,ferrie@link.net

\section{RÉFÉRENCES}

P. Bayley, Introduction, in PB., Cross-Cultural Perspectives on Parliamentary Discourse, Amsterdam/Philadelphia, John Benjamins, 2004. 
C. Bevitori, Negotiating conflict: Interruptions in British and Italian parliamentary debates, in PB., Cross-Cultural Perspectives on Parliamentary Discourse, Amsterdam/Philadelphia, John Benjamins, 2004.

P. Brown \& S. C. Levinson, Politeness: Some Universals in Language Usage Cambridge, Cambridge University Press, 1987.

T. Carbó, Parliamentary discourse when things go wrong: Mapping histories, contexts, conflicts, in PB., Cross-Cultural Perspectives on Parliamentary Discourse Amsterdam/Philadelphia, John Benjamins, 2004.

B. Dupret, Le Jugement en action. Ethnométhodologie du droit, de la morale et de la justice en Egypte, Genève, Droz, 2006.

S. Eggins \& D. Slade, Analysing Casual Conversation Londres, Cassel, 1997.

J. Elster, L'usage stratégique de l'argumentation, in «Négociation», II, 2005, pp. 59-82.

J. Elster C. Offe \& U. K. Preuss, Institutional Design in Post-communist Societies: Rebuilding the Ship at Open Sea, Cambridge, Cambridge University Press, 1998.

J-N. Ferrié, Le régime de la civilité. Public et réislamisation en Egypte, Paris, Presses du CNRS, 2004.

J-N. Ferrié \& B. Dupret, Préférences et pertinences: analyse praxéologique des figures du compromis en contexte parlementaire. A propos d'un débat égyptien, in «Information sur les sciences sociales », II, 2004, n43, pp. 263-290.

H. Garfinkel, Ethnomethodology's Program: Working Out Durkheim's Aphorism, in A.Warfield Rawls, Lanham, Rowman \& Littlefield Publishers, 2002.

E. Goffman, Forms of Talk, Philadelphia, University of Philadelphia Press, 1981.

A. O. Hirschman, Exit, Voice and Loyalty: Response to Decline in Firms, Organizations and States, Cambridge, Harvard University Press, 1970.

A. O Hirschman, Défection et prise de parole. Théorie et applications, Paris, Fayard, 1995.

C. Ilie, Histrionic and agonistic features of parliamentary discourse Studies, in «Communication Sciences », I, 2003, n³, pp. 25-53.

A. al-Khani, La Syrie, entre démocratie et gouvernement personnel, 1948-1958 (en arabe), Beyrouth, Dar al-Nafa'is, 2004.

M. Komter, La construction de la preuve dans un interrogatoire de police, in « Droit et Société», 2001, $\mathrm{n}^{\circ} 48$, pp. 367-93.

I. Leudar \& J. Nekvapil, L'islam et la guerre contre le Terrorisme. Une semaine dans la vie d'un réseau dialogique, in $\mathrm{BD} \& \mathrm{~J}-\mathrm{NF}$., Médias, guerres et identités: Les pratiques communicationnelles de l'appartenance politique, ethnique et religieuse (à paraître en 2007).

I. Leudar V. Marsland \& J. Nekvapil, On Membership Categorization: 'Us', 'Them' and 'Doing Violence' in Political Discourse, in «Discourse \& Society », XV, 2004, n²-3, pp. 243-266.

E. Livingston, An Anthropology of Reading, Bloomington and Indianapolis, Indiana University Press, 1995.

J. Nekvapil \& I. Leudar, On Dialogical Networks: Arguments about the Migration Law in Czech Mass Media in 1993, in SH. \& WH., Language, Interaction and National Identity, Aldershot, Ashgate, 2002.

E. Schegloff, Between Macro and Micro: Contexts and Other Connections, in J.A R.M.BG. \& NS., The Micro-Macro Link, Berkeley, University of California Press, 1987.

T. Van Dijk, Teun, Contextualization in Parliamentary Discourse : Aznar, Iraq and the Pragmatics of Lying, in Congreso Discurso Oral Almeria, 24-25 novembre 2005.

R. D. Watson, Formulations as Conversational Objects, in GP., Everyday Language. Studies in Ethnomethodology, New York, Irvington, 1979. 